\title{
Nanotheranostics
}

2019; 3(1): 66-88. doi: 10.7150/ntno.30052

Review

\section{Biological Effects of Nanoparticles on Macrophage Polarization in the Tumor Microenvironment}

\author{
Derek Reichel ${ }^{1}$, Manisha Tripathi ${ }^{1,4}$ and J. Manuel Perez ${ }^{1,2,3}{ }^{凶}$ \\ 1. Department of Neurosurgery, Cedars-Sinai Medical Center, Los Angeles, CA 90048 \\ 2. Biomedical Imaging Research Institute, Cedars-Sinai Medical Center, Los Angeles, CA 90048 \\ 3. Samuel Oschin Comprehensive Cancer Institute, Cedars-Sinai Medical Center, Los Angeles, CA 90048 \\ 4. Current Address: Department of Cell Biology and Biochemistry, Texas Tech University Health Sciences Center, Lubbock, TX 79430, USA \\ $\triangle$ Corresponding author: J Manuel Perez, PhD. Department of Biomedical Sciences \& Department of Neurosurgery, Biomedical Imaging Research Institute \& \\ Samuel Oschin Comprehensive Cancer Institute, Cedar Sinai Medical Center, 127 S. San Vicente Blvd, Suite A8113, Los Angeles CA, 90048. Email: \\ jmanuel.perez@cshs.org
}

(C) Ivyspring International Publisher. This is an open access article distributed under the terms of the Creative Commons Attribution (CC BY-NC) license (https://creativecommons.org/licenses/by-nc/4.0/). See http://ivyspring.com/terms for full terms and conditions.

Received: 2018.09.18; Accepted: 2018.12.02; Published: 2019.01.01

\begin{abstract}
Biological interactions between tumor-associated macrophages (TAMs), cancer cells and other cells within the tumor microenvironment contribute to tumorigenesis, tumor growth, metastasis and therapeutic resistance. TAMs can remodel the tumor microenvironment to reduce growth barriers such as the dense extracellular matrix and shift tumors towards an immunosuppressive microenvironment that protects cancer cells from targeted immune responses. Nanoparticles can interrupt these biological interactions within tumors by altering TAM phenotypes through a process called polarization. Macrophage polarization within tumors can shift TAMs from a growth-promoting phenotype towards a cancer cell-killing phenotype that predicts treatment efficacy. Because many types of nanoparticles have been shown to preferentially accumulate within macrophages following systemic administration, there is considerable interest in identifying nanoparticle effects on TAM polarization, evaluating nanoparticle-induced TAM polarization effects on cancer treatment using drug-loaded nanoparticles and identifying beneficial types of nanoparticles for effective cancer treatment. In this review, the macrophage polarization effects of nanoparticles will be described based on their primary chemical composition. Because of their strong macrophage-polarizing and antitumor effects compared to other types of nanoparticles, the effects of iron oxide nanoparticles on macrophages will be discussed in detail. By comparing the macrophage polarization effects of various nanoparticle treatments reported in the literature, this review aims to both elucidate nanoparticle material effects on macrophage polarization and to provide insight into engineering nanoparticles with more beneficial immunological responses for cancer treatment.
\end{abstract}

Key words: Nanoparticles, Macrophage polarization, Tumor microenvironment, Cancer, Iron oxide

\section{Introduction}

The tumor microenvironment is a unique biological environment that promotes tumorigenesis, tumor metastasis and therapeutic resistance [1-4]. Interactions between different cell populations within tumors contribute to the formation and proliferation of the tumor microenvironment [5], and tumor-associated macrophages (TAMs) are a major constituent within tumors [6]. Although the biological origins of TAMs are not fully understood, experimental evidence suggests that TAMs are a heterogeneous group of cells derived from both tissue-resident macrophages and bone marrow-derived monocytes [7, 8]. While tissue-resident macrophages support cell growth, destroy foreign invaders and protect healthy tissues from aberrant remodeling, TAMs aid tumor 
development, demonstrate suppressed immune activity and assist in tissue remodeling. [9]. Figure 1 describes some of the interactions between macrophages and other types of cells within tumors. TAMs secrete growth factors such as vascular endothelial growth factor A (VEGF-A) or placental growth factor (PGF) that can increase blood vessel formation by tumor endothelial cells [10]. These new blood vessels increase nutrient delivery to cancer cells and enhance cancer cell growth within dense tumors. In addition to secreting growth factors, TAMs also secrete enzymes such as matrix metalloproteinase-9 (MMP-9), serine proteases and cathepsins that can degrade the extracellular matrix (ECM), facilitate tumor invasion to adjacent organs, and promote metastasis [11, 12]. Both adipose cells and fibroblasts increase macrophage recruitment into tumors, which amplifies their microenvironment-remodeling capabilities and further promotes tumor invasion and metastasis [13, 14]. TAMs also enhance the survival, migration and chemoresistance of cancer-associated stem cells, which may allow tumors to regrow following otherwise successful treatments [15]. Recent reports suggest that TAMs can suppress Natural Killer (NK) and $\mathrm{T}$ cell activity within tumors by preventing immune cells from properly identifying cancer cells [16, 17], which can hinder targeted immune responses to damaged cancer cells and reduce chemotherapy efficacy. Because TAMs contribute to many interactions within tumors that form the tumor microenvironment, TAMs are considered to be a promising therapeutic target for cancer treatment [18].

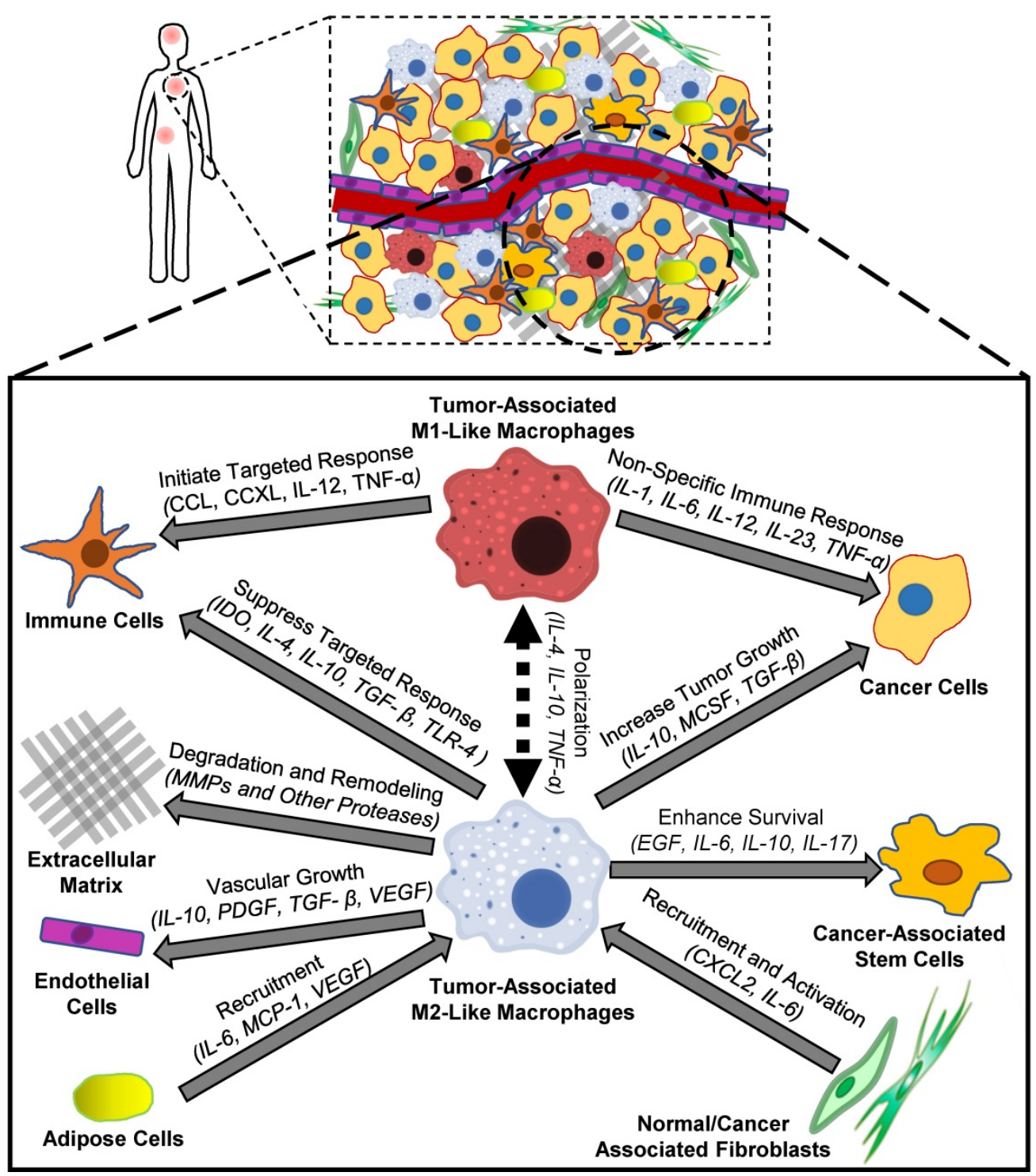

Figure 1. Biological effects of macrophages on cellular components of the tumor microenvironment. Tumor-associated M2-like macrophages contribute to tumor microenvironment remodeling by removing growth barriers such as the extracellular matrix and promoting vascular growth. In addition, tumor-associated M2-like macrophages promote an immunosuppressive tumor microenvironment that protects cancer cells from targeted immune responses. Following polarization towards an Ml-like state, these macrophages can initiate both non-specific and targeted immune responses towards cancer cells. 
Macrophages themselves can undergo significant phenotypic changes in response to extracellular signals or foreign entities, such as macromolecules or bacteria, through a process called polarization [19]. Initially, many researchers hypothesized that macrophage polarization divided macrophages into two distinct subtypes: type 1 macrophages (M $\varphi 1)$ and type 2 macrophages $(\mathrm{M} \varphi 2)$ [20]. $\mathrm{M} \varphi 1$ were described as pro-inflammatory, antibacterial macrophages because they are often found at sites of inflammation or bacterial infection. Both obesity-induced inflammation in adipose tissue and insulin resistance have been attributed to $\mathrm{M} \varphi 1$ $[21,22]$. In addition, increased $M \varphi 1$ prevalence in cancer patients could also contribute to immune-related adverse events from immune checkpoint inhibitor therapies, which can be difficult to manage clinically [23]. In contrast, $\mathrm{M} \varphi 2$ were classified as anti-inflammatory macrophages because of their presence at wound sites following injury [24]. However, recent studies have shown that macrophage polarization is more accurately described as shifts toward an idealized M1 or M2 polarization state that results in a spectrum of various macrophage phenotypes $[25,26]$. For simplicity, this review will refer to any macrophages with M1-like phenotypes as M1-like macrophages and any macrophages with M2-like phenotypes as M2-like macrophages. This paradigm has also been used to describe the behavior of TAMs [27, 28]. M1-like macrophages within tumors can identify cancer cells and initiate targeted immune responses, while M2-like macrophages within tumors can promote tumor growth and proliferation [29]. Cancer researchers typically consider TAMs within patients to consist of predominantly M2-like macrophages [30], and treatments targeting M2-like macrophages within tumors have shown promise to improve cancer treatment. Specifically, Georgoudaki et al. demonstrated that the treatment of tumors with M2-like macrophage-targeting antibodies increased the prevalence of M1-like macrophages within tumors and promoted a T-cell dependent immune response that decreased tumor size, prevented cancer progression and inhibited metastasis in mouse models [31].

Recent evidence suggests that nanoparticle uptake can contribute to macrophage polarization within tumors $[32,33]$. Nanoparticles are defined as small $(<200 \mathrm{~nm}$ diameter) particles comprised of metals, polymers or other materials that often contain hydrophobic regions enveloped by a hydrophilic shell. Because these hydrophobic regions can entrap hydrophobic drugs, nanoparticle delivery of hydrophobic anticancer drugs to tumors has gathered significant interest as a cancer treatment approach [34,
35]. Nanoparticles have been recognized to localize within both primary and metastatic tumors following systemic administration, and the biological factors leading to this accumulation profile have been thoroughly studied [36-38]. More specifically, nanoparticles have been shown to accumulate within both TAMs and healthy macrophages following systemic administration [39, 40]. Previous investigations have suggested that the sustained release of drugs from nanoparticle-containing TAMs into the surrounding cancer cells can alter the therapeutic efficacy of drug-loaded nanoparticles [41, 42]. Specifically, Miller et al. demonstrated that the accumulation of platinum prodrug-loaded polymeric nanoparticles within tumor-associated macrophages alters the spatial distribution of drug within tumors and controls nanoparticle treatment efficacy, while the depletion of macrophages from tumors reduces nanoparticle treatment efficacy [42]. However, this review will focus more on the nanoparticle effects on macrophage polarization, rather than macrophage effects on nanoparticle drug delivery to tumors. By comparing the macrophage polarization effects of various nanoparticle treatments to each other, the effect of nanoparticle material composition on macrophage polarization can be elucidated, which may provide design considerations for drug-loaded nanoparticle formulations.

\section{Measurement of Macrophage Polarization}

Because the presence of M2-like macrophages within tumors is correlated with poor patient prognosis in several types of cancer [43-45], there is interest in both quantifying macrophage polarization states in tumors and determining the polarization effects of nanoparticles within tumors. Macrophage polarization states are often measured using three main cell properties: cell surface protein expression, enzymatic activity and cytokine secretion. Table $\mathbf{1}$ summarizes some of the most common markers for macrophage polarization that increase in response to the indicated polarization state, and indicates some characterization methods for these markers that are described in the literature [46-50]. Because these markers can be affected by multiple biological factors and are time-dependent, researchers often measure multiple M1 and M2 polarization markers states at several timepoints. It should be noted that there is no standardized protocol to quantify macrophage polarization, and research groups often select various markers and detection timepoints based on their experience. In addition, researchers often use various nanoparticle treatment conditions to observe polarization effects. As a result, research groups often report inconsistent or contradictory results, and 
additional experiments are needed to quantitatively compare the biological effects of nanoparticles on macrophage polarization.

\section{Macrophage Responses to Nanoparticles}

Macrophage responses to nanoparticles can depend on factors such as dose, route of administration, size, nanoparticle composition and nanoparticle surface properties. Macrophages in patients are typically exposed to nanoparticles in three main ways: 1 ) oral or parenteral administration of nanoparticle-based pharmaceutical formulations, 2) inhalation of airborne nanoparticles from pollution or occupational exposure, or 3) generation of nanoparticles in the body due to the degradation of metallic implants. Once nanoparticles enter the body, macrophages can identify nanoparticles as foreign bodies due to surface opsonization and take them up by endocytosis or phagocytosis [51]. Because these materials are not recognized as inert, macrophages will often respond to their uptake by undergoing polarization. The exact contributions of specific nanoparticle properties, such as material type, core and shell composition, therapeutic payload, or shape, to their overall macrophage polarization properties are not fully understood. Because nanoparticles are often described in the literature based on their core composition, nanoparticles in this review are categorized based on their core material rather than size or surface charge. Based on this classification system, eight main types of nanoparticles were identified: silica, gold, polymeric, cationic polymer, liposome, carbon, metallic and iron oxide. These nanoparticle types represent both nanoparticles in clinical trials or in the environment due to pollution. Table 2 summarizes the effects of nanoparticle treatment on macrophage polarization markers that will be described in detail in the following sections. Some of the nanoparticle effects on specific polarization markers could not be identified from the literature, indicating the need for additional macrophage polarization characterization experiments.

In order to evaluate the significance of nanoparticle-induced macrophage polarization effects, they must be considered in comparison to small molecule-induced macrophage polarization effects. The immunological effects of anticancer drugs such as doxorubicin or paclitaxel within tumors have been investigated for many years [52-54]. Although some anticancer drugs have been shown to suppress immune activity within tumors and increase tumor growth [53], many of these drugs remain in clinical use for cancer treatment. Macrophages are often polarized in vitro by treatment with biomolecules such as interferon- $\gamma$ (IFN- $\gamma$ ), IL-4, IL-10 or lipopolysaccharide (LPS). By comparing the polarization of nanoparticle-treated macrophages to either drug- or biomolecule-treated macrophages, researchers can gain a more detailed understanding of nanoparticle-induced macrophage polarization. Towards this goal, significant efforts are needed to identify reference treatment conditions for accurate comparisons between small molecule- and nanoparticle-induced effects.

Table 1. Macrophage polarization markers. Markers increase expression or secretion in response the indicated polarization state.

\begin{tabular}{|c|c|c|c|c|}
\hline Marker & Polarization State & Detection Methods & Type & Reference \\
\hline IL-1 $\beta$ & M1-Like & ELISA/PCR & Cytokine & {$[48,170,171]$} \\
\hline IL-6 & M1-Like & & Cytokine & {$[48,171,172]$} \\
\hline IL-10 & M2-Like & & Cytokine & {$[48,50,170-172]$} \\
\hline IL-12 & M1-Like & & Cytokine & {$[48,50,170,172]$} \\
\hline IL-23 & M1-Like & & Cytokine & {$[50]$} \\
\hline TGF- $\beta$ & M2-Like & & Cytokine & {$[48,171-173]$} \\
\hline TNF-a & M1-Like & & Cytokine & {$[48,170-172]$} \\
\hline CD68 & M1-Like & FACS/IHC & Cell Surface Protein & {$[170,172,173]$} \\
\hline CD80 & M1-Like & & Cell Surface Protein & {$[170,173]$} \\
\hline CD86 & M1-Like & & Cell Surface Protein & {$[170,172]$} \\
\hline CD163 & M2-Like & & Cell Surface Protein & {$[48,49,170,172]$} \\
\hline CD206 & M2-Like & & Cell Surface Protein & {$[170,172]$} \\
\hline Ferroportin & M2-Like & & Cell Surface Protein & {$[149,153]$} \\
\hline Reactive Oxygen Species (ROS) & M1-Like & Fluorescence-Based Assays & Enzymatic Product & {$[171]$} \\
\hline Nitric Oxide & M1-Like & & Enzymatic Product & {$[49,50,170-173]$} \\
\hline Arginase & M2-Like & IHC/Western Blot & Enzyme & {$[49,50,172,173]$} \\
\hline Ferritin & M1-Like & & Cell Protein & {$[149,153]$} \\
\hline Inducible Nitric Oxide Synthase (iNOS) & M1-Like & & Enzyme/Enzymatic Product & {$[49,50,170-173]$} \\
\hline Transferrin Receptor 1 & M2-Like & & Cell Surface Protein & [149] \\
\hline
\end{tabular}


Table 2. Effects of nanoparticle treatment on macrophage cell surface protein expression. Size range refers to the size of the nanoparticles as reported in the references. Because of their inconsistent effects, biological-based nanoparticles were excluded from the table.

\begin{tabular}{|c|c|c|c|c|c|c|c|c|c|c|c|}
\hline & & & M1 Markers & & & & M2 Mark & cers & & & \\
\hline Nanoparticle & Overall & Size & CD68/CD80/ & IL-1 $\beta /$ IL-6/IL-12/ & iNOS/NO & ROS & CD163/ & IL-10 & TGF- $\beta$ & Arginase-1 & Reference \\
\hline Type & $\begin{array}{l}\text { Polarization } \\
\text { Effect }\end{array}$ & $\begin{array}{l}\text { Range } \\
(\mathrm{nm})\end{array}$ & CD86 & IL-23/TNF-a & & Generation & CD206 & & & & \\
\hline Silica & M1-Like & $10-1000$ & No Change & Increase & Increase & Increase & - & $\begin{array}{l}\text { No } \\
\text { Change }\end{array}$ & Increase & - & [59-64] \\
\hline Gold & M1-Like & $10-300$ & No Change & Increase & Increase & Increase & - & Decrease & - & - & {$[60,70-73]$} \\
\hline Polymeric & M2-Like & $30-600$ & Decrease & Decrease & Decrease & Decrease & Increase & Increase & Decrease & Increase & {$[77-80]$} \\
\hline $\begin{array}{l}\text { Cationic } \\
\text { Polymer }\end{array}$ & M1-Like & $110-22000$ & Increase & Increase & Increase & Increase & Decrease & Decrease & Decrease & Increase & [85-93] \\
\hline Liposome & M2-Like & $70-400$ & - & Decrease & $\begin{array}{l}\text { No } \\
\text { Change }\end{array}$ & No Change & Increase & Increase & - & Increase & {$[96,98,99]$} \\
\hline Carbon & M1-Like & $70-70000$ & Increase & Increase & Increase & Decrease & Increase & Increase & $\begin{array}{l}\text { No } \\
\text { Change }\end{array}$ & Increase & [104-111] \\
\hline Metallic & M1-Like & $20-200$ & Increase & Increase & Increase & Increase & Decrease & Increase & - & Increase & $\begin{array}{l}{[126-129,136,137,} \\
139,140]\end{array}$ \\
\hline Iron Oxide & M1-Like & $30-280$ & Increase & Increase & Increase & Increase & Decrease & Increase & - & Decrease & $\begin{array}{l}{[150,151,154,155,} \\
161,162,165,174]\end{array}$ \\
\hline
\end{tabular}

\section{Silica Nanoparticles}

Partially porous silica-based nanoparticles called mesoporous silica nanoparticles (MSNs) have been investigated as PET imaging devices entrapping radioisotopes such as copper-64 or zirconium- 89 [55, 56]. Clinical trials have been performed to assess the lymph node mapping and photothermal therapy capabilities of these nanoparticles [57, 58]. Because of their widespread use in medical fields, MSNs with a variety of sizes have been prepared, and the size effects of MSNs on macrophage polarization have been investigated. For example, Kwon et al. measured the treatment effects of 100-200 nm diameter MSNs on macrophage polarization [59]. Smaller diameter MSN treatments increased ROS generation in macrophages compared to larger diameter MSN treatments. This observation suggests that the surface area-to-volume ratios of MSNs control their ROS generation potential. Furthermore, none of the MSNs in this study affected IL-6 or TNF-a secretion from macrophages. In contrast, Bancos et al. found that treatment with $10 \mathrm{~nm}$ but not $300 \mathrm{~nm}$ diameter MSNs increased TNF-a secretion from macrophages after $24 \mathrm{~h}$ [60]. Smaller diameter MSNs in this study demonstrated greater intracellular accumulation than larger diameter MSNs following macrophage treatment, suggesting that the intracellular accumulation of MSNs causes TNF-a secretion. However, the MSN treatments in this study did not affect CD80, CD86 or CD40 expression on macrophages. Other research groups observed that the treatment of macrophages with silica-based microparticles (1-100 $\mu \mathrm{m}$ diameter) had no effect on either IL-6 or IL-10 secretion [61]. In a different study, Park et al. found that MSN treatments increased IL-1, IL-6, TNF- $\alpha$ and iNOS secretion from peritoneal macrophages isolated form mice [62]. Together, these results suggest that smaller diameter MSNs promote greater M1 marker cytokine secretion from macrophages than larger diameter MSNs or microparticles.

In order to elucidate the contributions of surface area and diameter to the silica nanoparticle size effects on macrophage polarization, Kusaka et al. prepared a silica particle library with diameters between 30 and $10000 \mathrm{~nm}$, and then measured IL-1 $\beta$ secretion from silica particle-treated macrophages as a polarization marker [63]. The group further investigated the mechanism of size-dependent IL-1 $\beta$ secretion, and a summary of their findings is in Figure 2. The smaller diameter MSNs had stronger effects on IL-1 $\beta$ secretion than larger diameter MSNs, even after accounting for differences in their surface area-to-volume ratios (Figure 2A). Macrophage treatment with the microfilament inhibitor cytochalasin D (Cyto D) prevented intracellular uptake of the silica particles, as indicated by the cell surface marker CD11b (Figure 2B). Because Cyto D pretreatment of macrophages mitigated IL-1 $\beta$ secretion increases, they concluded that silica particle uptake via a cytoskeleton-dependent pathway is necessary for IL-1 $\beta$ secretion (Figure 2C). The pretreatment of macrophages with the lysosomal acidification inhibitor balfilomycin $\mathrm{A}$ also mitigated potential increases in IL-1 $\beta$ secretion, indicating that silica particle-induced lysosomal damage may also contribute to IL-1 $\beta$ secretion increases (Figure 2D). Taken together, these experiments suggest that larger diameter MSNs and silica microparticles have minimal macrophage polarization effects due to decreased intracellular accumulation and minimal lysosomal disruption.

Surface modifications to MSNs have also been investigated as a method to modulate their 
macrophage polarization properties. Wan et al. prepared MSNs that were coated with human plasma proteins [64]. By treating these MSNs with deglycosylases, the surface glycosylation of these MSNs could be modulated without altering their protein coating. Regardless of their surface glycosylation state, these MSNs increased IL-1 $\beta$ secretion, decreased TGF- $\beta$ secretion, and had no effect on TNF-a secretion from M1-like macrophages. Both MSN treatments also increased TNF-a secretion from M2-like macrophages but had no effect on IL-1 $\beta$ or TGF- $\beta$ secretion. However, surface glycosylation of
MSNs reduced the magnitude of the observed M1 polarization effects. In another study, macrophage treatment with TAT peptide-functionalized or bovine serum albumin-functionalized MSNs was found to increase ROS generation in macrophages compared to unmodified MSN treatments due to increased cellular uptake [65]. Taken together, these reports indicate that increasing the size of silica particles can reduce their cellular uptake and minimize their M1 polarizing capabilities, and surface modifications to MSNs can further control their cellular uptake and modulate their polarization effects.
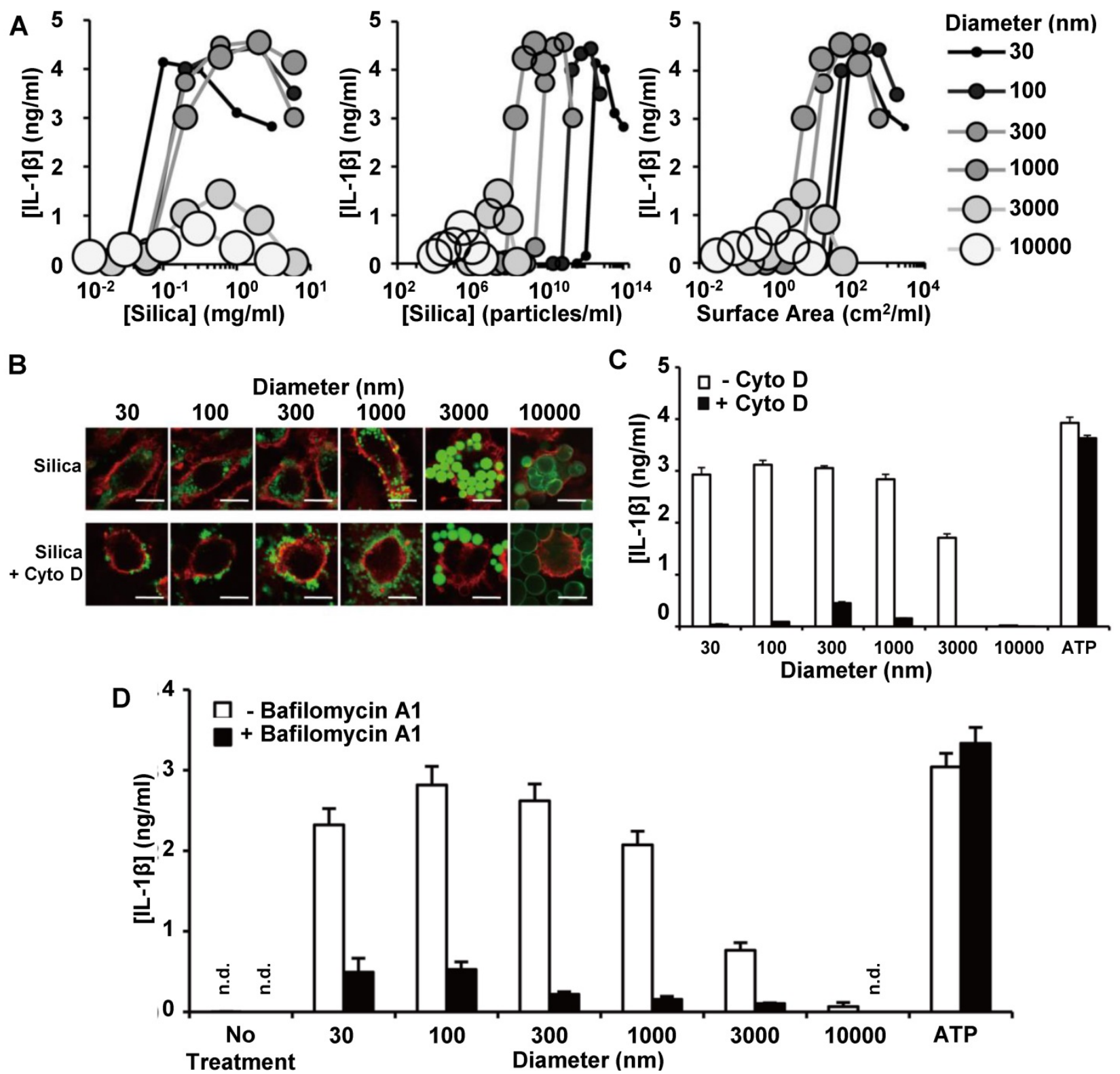

Figure 2. IL-1 $\beta$ secretion from macrophages treated with silica particles of various diameters. IL- $1 \beta$ secretion is graphed as a function of mass-based (left), mole-based (middle) or surface area-based (right) concentration (A). Macrophage uptake of FITC-labelled silica particles (green) with and without Cyto D pre-treatment. Red indicates CDI 1b, while green indicated silica particles (B). Quantification of IL-1 $\beta$ secretion from macrophages following silica particle treatment with and without Cyto D pre-treatment. (C). Quantification of IL-1 $\beta$ secretion from macrophages following silica particle treatment with and without bafilomycin AI pre-treatment (D). ATP treatment was used as a control for potential cytotoxicity-induced effects on IL-1 $\beta$ secretion in (C) and (D). Adapted from [63] with permission. 
A

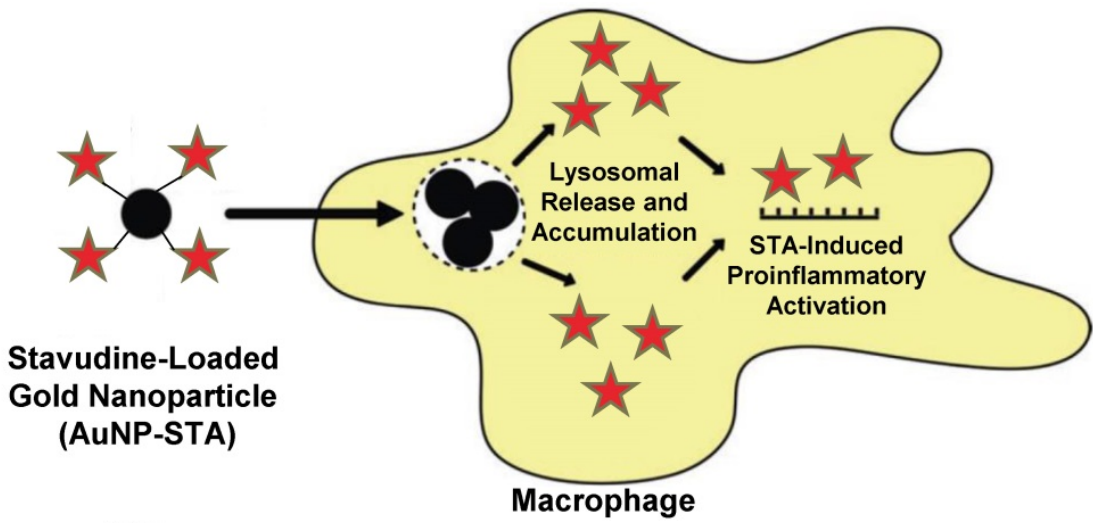

B
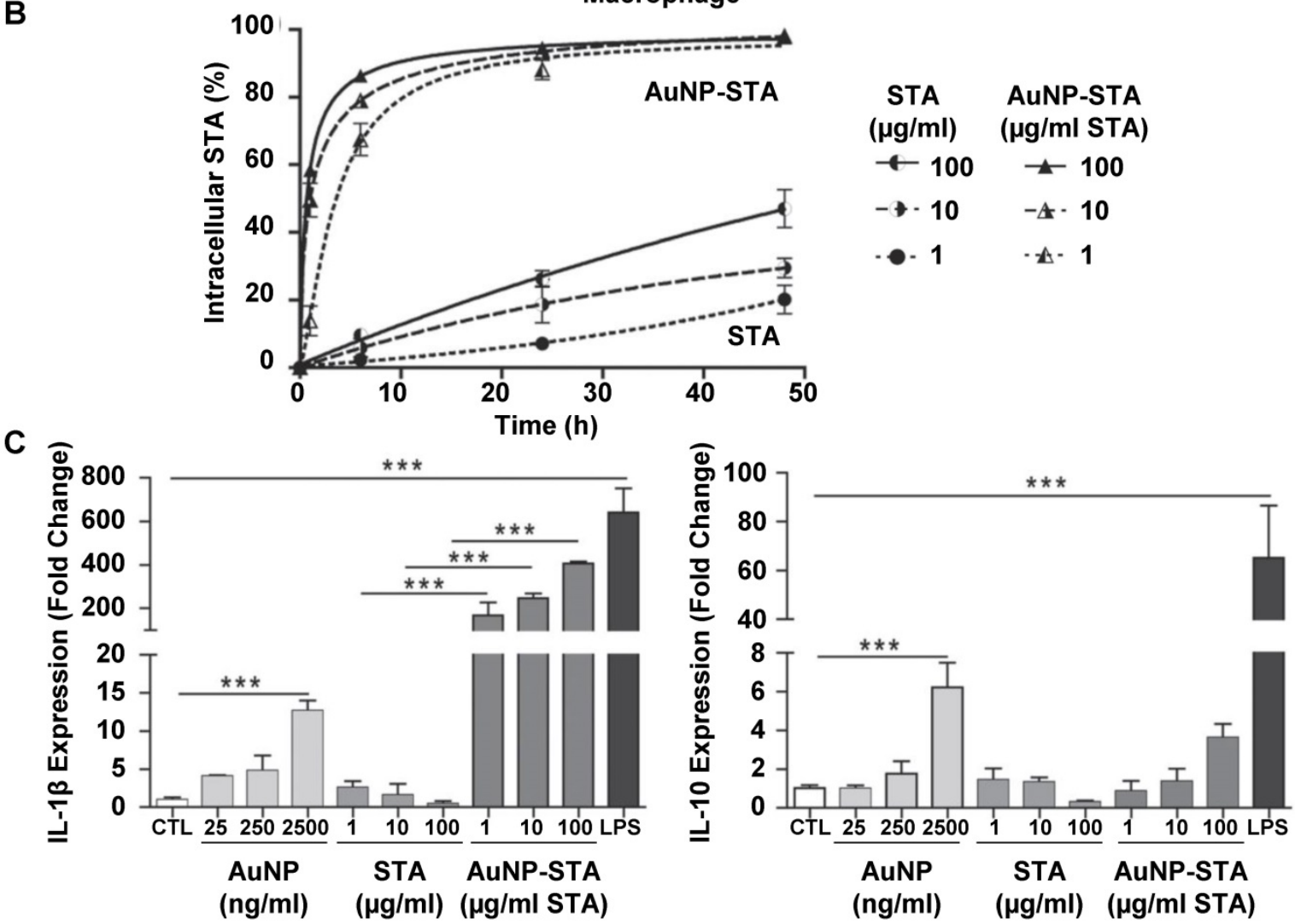

Figure 3. General mechanism for stavudine-loaded gold nanoparticle-induced macrophage polarization. Gold nanoparticles were mixed with STA to prepare AuNP-STA, which is expected to release drug inside macrophages following uptake (A). Uptake of STA in macrophages following treatment with either stavudine-complexed gold nanoparticles or free stavudine (B). IL-1 13 and IL-10 secretion from macrophages treated with either gold nanoparticles, stavudine or stavudine-complexed gold nanoparticles (C). Adapted with permission from [70].

\section{Gold Nanoparticles}

Gold nanoparticles have been investigated extensively in pre-clinical and clinical studies as tools for drug delivery, diagnostics and biomedical imaging due to their optical, acoustic and Raman properties [66-69]. In one study, gold nanoparticles were developed as a delivery system for the antiretroviral drug stavudine (STA) [70]. Figure 3 summarizes the findings of this study. Stavudine-loaded gold nanoparticles (AuNP-STA) were prepared by mixing gold nanoparticles with stavudine in water. These AuNP-STA nanoparticles were expected to accumulate within macrophages and release STA into cells, which would induce proinflammatory macrophage activation and improve drug efficacy (Figure 3A). Indeed, nanoparticle complexation of STA increased its uptake into macrophages compared to free STA treatments (Figure 3B). In addition, stavudine complexation did not affect the uptake of gold nanoparticles. The treatment of macrophages with empty gold nanoparticles increased both IL-1 $\beta$ and IL-10 secretion, while free stavudine treatment had no effect on these markers (Figure 3C). Surprisingly, treatment with stavudine-complexed gold nanoparticles drastically increased IL-1 $\beta$ secretion from 
macrophages compared to either individual treatment. In addition, stavudine-complexed gold nanoparticle treatments increased IL-10 secretion from macrophages, suggesting that nanoparticle complexation of stavudine enhances its action in macrophages. These findings highlight a potential strategy for drug-loaded nanoparticles to improve the treatment efficacy of antiretroviral drugs by targeting macrophages, increasing macrophage uptake of drug-loaded nanoparticles compared to free drug, and modulating macrophage activity to promote an effective immune response towards viruses.

Several researchers have observed changes in cytokine secretion profiles from macrophages following treatment with gold nanoparticles. Bastus et al. demonstrated that the treatment of macrophages with amyloid growth inhibitory peptide- or sweet arrow peptide-conjugated gold nanoparticles increased TNF- $\alpha$, IL-1 $\beta$ and IL-6 secretion due to interactions between peptide-conjugated nanoparticles and toll-like receptor 4 [71, 72]. Nanoparticle-treated macrophages in this study also demonstrated increased iNOS expression as part of their immune response. In another study, Pal et al. measured the effects of citrate-coated gold nanoparticle treatment on tumor-associated macrophages from tumor-bearing mice, splenic macrophages from tumor-bearing mice and macrophages from healthy mice [73]. ROS generation increased more in tumor-associated macrophages than in splenic macrophages from tumor-bearing mice or macrophages from healthy mice. In addition, gold nanoparticle treatments decreased both TNF-a and IL-10 secretion from tumor-associated macrophages while also increasing IL-12 secretion. The contradictory cytokine secretion observations between the two previously discussed reports may be attributed to differences between citrate and peptide surface coatings of gold nanoparticles; however, elucidating the effects of nanoparticle surface coating on macrophage polarization while keeping other factors such as surface charge constant remains difficult. These results indicate the significance of nanoparticle surface coatings to macrophage polarization, and further emphasize the need to measure multiple polarization markers to accurately characterize macrophage polarization.

\section{Polymeric Nanoparticles}

Polymeric nanoparticles with a core-shell structure that are prepared from biocompatible polymers such as poly(lactic-co-glycolic acid) (PLGA) or polylactic acid have been used as biocompatible drug delivery systems for the controlled release of a variety of drugs [74-76]. Because the chemical properties of polymers can be finely tuned during their synthesis, the physical-chemical properties of polymeric nanoparticles can be finely tuned in comparison to other types of nanoparticles by using libraries of selected polymers with desired properties during nanoparticle preparation. By developing and testing several polymeric nanoparticles with varying chemical properties, the contributions of specific physical-chemical nanoparticle properties to macrophage polarization can be elucidated. Towards this goal, Ye et al. prepared polymers consisting of starch and octanoic acid, and then further modified these polymers with sulfobetaine groups in order to prepare either uncharged or zwitterionic nanoparticles [77]. By treating macrophages with these nanoparticles, the group could investigate the effects of protein binding to the nanoparticle surface, also called opsonization, on macrophage polarization. Although the uncharged surface nanoparticle increased in size and gained a negative surface charge during storage in $10 \%$ serum due to protein binding, zwitterionic nanoparticles remained stable in $10 \%$ serum. As a result of protein binding, the uncharged nanoparticles demonstrated greater accumulation in macrophages than the zwitterionic nanoparticles. The uncharged nanoparticles increased IL-6 and TNF- $\alpha$ secretion from macrophages in comparison to zwitterionic nanoparticles, presumably due to the presence of serum proteins on the nanoparticle surface. Based on these results, the group hypothesized that the opsonization of polymeric nanoparticles contributes greatly to their macrophage uptake and polarization effects. In addition to opsonization-induced macrophage interactions, sugar groups can also induce macrophage interactions. Su et al. developed polymeric nanoparticles with various sugar groups in the corona, in order to target macrophage surface receptors such as CD206 (mannose receptor) [78]. Macrophage treatment with these nanoparticles increased CD86 expression, while also decreasing CD23 and CD206 expression. These nanoparticles also increased IL-12, MCP-1 and TNF- $\alpha$ secretion from macrophages, while also decreasing IL-10 secretion. It should be noted that the polyethylene glycol (PEG)-modified nanoparticles used in this study had no effect on macrophage polarization, presumably because they were not taken up by macrophages. Together, these results indicate that increasing the surface ionization or adding inert polymers to the surface of nanoparticles to reduce their opsonization and prevent specific nanoparticle-macrophage interactions can reduce their macrophage polarization capabilities.

To elucidate the effects of specific polymeric nanoparticle surface properties on macrophage 
polarization, Wang et al. prepared a library of 13 polymeric nanoparticles with different functional groups on the surface and characterized their macrophage polarization properties [79]. These nanoparticles had minimal differences in surface charge or melting temperature. The surfaces of these nanoparticles were characterized using a 22-parameter polymer property model, and both IL-10 and TNF-a secretion was measured from nanoparticle-treated macrophages. To quantify the specific effects of nanoparticle chemical properties on macrophages, the group developed mathematical models relating IL-10 and TNF- $\alpha$ secretion to nanoparticle surface properties. These mathematical models, also called quantitative structure-activity relationships (QSARs), indicated that hydrogen bond donors and acceptors on the nanoparticle surface predicted cytokine secretion from macrophages. The group speculated that hydrogen bond donors and acceptors on the nanoparticle surface facilitated opsonization, which caused macrophage polarization following uptake. In additional work by this group, QSARs were developed to predict in vivo macrophage polarization [80]. Figure 4 highlights some of the key results from this study. First, the group treated mice with various nanoparticles and isolated macrophages from these mice. Next, the group characterized TNF- $\alpha$ secretion from these macrophages (Figure 4A). Then, IL-10 secretion was measured from these macrophages (Figure 4B). After that, the ratio of arginase to iNOS activity was measured (Figure 4C). Using these measured data, QSARs were developed to predict cytokine secretion or enzymatic activity in macrophages. These QSARs identified key physical and chemical properties of polymeric nanoparticles that contributed to in vivo macrophage polarization. In addition, the QSARs demonstrated that specific polymer properties affect each macrophage polarization marker differently, and that the surface properties of a polymeric nanoparticle must be considered carefully when controlling the macrophage polarization properties of a polymeric nanoparticle. Overall, these findings indicate that polymeric nanoparticle surface properties affect each macrophage polarization marker differently, and polymeric nanoparticles must be carefully designed to control their polarization properties based on several markers.

\section{Cationic Polymers}

Cationic polymers have been used to prepare nanoparticle delivery systems for both nucleic acids and anionic drugs by charge neutralization [81-83]. Depending on the chemical structures of the polymer and payload, the cationic groups in these nanoparticles can either be free, exposed on the nanoparticle surface or concealed in the nanoparticle core. Unlike other polymeric nanoparticles that are typically developed with inert polymers, cationic polymers have been shown to cause toxicity by interacting with cellular components such as the mitochondria [84]. As a result, the biological effects of cationic polymer-containing nanoparticles are often considered to be distinct from neutral or anionic polymeric nanoparticles. Treatment of macrophages with cationic polymers such as polyethyleneimine (PEI) or cationic dextran has been demonstrated to induce M1-like polarization through toll-like receptor 4 (TLR4) signaling and promote immune cell infiltration into tumors [85]. In another study, Lunov et al. demonstrated that the treatment of macrophages with cationic polystyrene nanoparticles increased IL-1 $\beta$ secretion and promoted inflammasome formation [86]. In contrast, macrophage treatment with non-ionic or anionic polystyrene nanoparticles in this study had no effect on these markers. Regardless of their surface functionalization, polystyrene nanoparticle treatments did not affect TNF-a secretion from macrophages. To assess the role of ROS generation in macrophage polarization, the group loaded the nanoparticles with ROS scavengers and then treated macrophages. Because these nanoparticles had minimal effects on macrophage polarization, the polarization effects of cationic polystyrene nanoparticles were concluded to be ROS-mediated. However, other reports found that both cationic and anionic polystyrene nanoparticle treatments of M2-like macrophages decreased CD163 and CD200R expression without affecting CD86 expression [87]. The treatment of M2-like macrophages with either nanoparticle also decreased IL-10 secretion without affecting TNF-a secretion, IL-1 $\beta$ secretion, or NOS2 expression. Together, these results suggest that cationic groups on the surface of nanoparticles can induce M1-like polarization in macrophages.

In addition to the charged groups on the surface, polymeric nanoparticles can also conceal charged groups in the core. Castro et al. used the cationic carbohydrate chitosan and the anionic peptide poly- $\gamma$-glutamic acid to prepare nanoparticles and measured their effects on macrophage polarization [88]. Macrophages that were treated with these nanoparticles demonstrated increased CD40, CD86 and HLA-DR expression. In addition, these nanoparticles increased IL-12, IL-23, TNF-a, IL-6 and IL-10 secretion from macrophages, while also preventing IL-10-induced M2-like polarization. Goncalves et al. confirmed the effects of chitosan/poly- $\gamma$-glutamic acid nanoparticle 
treatments on macrophages [89]. In further experiments, Goncalves et al. demonstrated that loading the anti-inflammatory drug diclofenac into these nanoparticles mitigated their effects on IL-12, IL-23 and TNF-a secretion from macrophages. In a different study, Ma et al. also demonstrated that chitosan nanoparticle treatments increased IL-1, IL-6 and TNF-a secretion from macrophages [90]. However, the treatment of macrophages with arginine-based polyester amide/hyaluronic acid nanoparticles increased IL-10 secretion, TNF-a secretion and arginase expression [91]. It should be noted that the nanoparticles in this study contained a molar excess of hyaluronic acid, and charge neutralization due to ionic bonding could mitigate the M1-polarizing effects of the cationic arginine-based polyester amide polymer in these nanoparticles. Taken together, these results suggest that concealing or neutralizing charged groups on cationic polymers in the core of a nanoparticle does not mitigate their M1 polarizing capabilities, probably due to nanoparticle dissociation following cellular uptake.
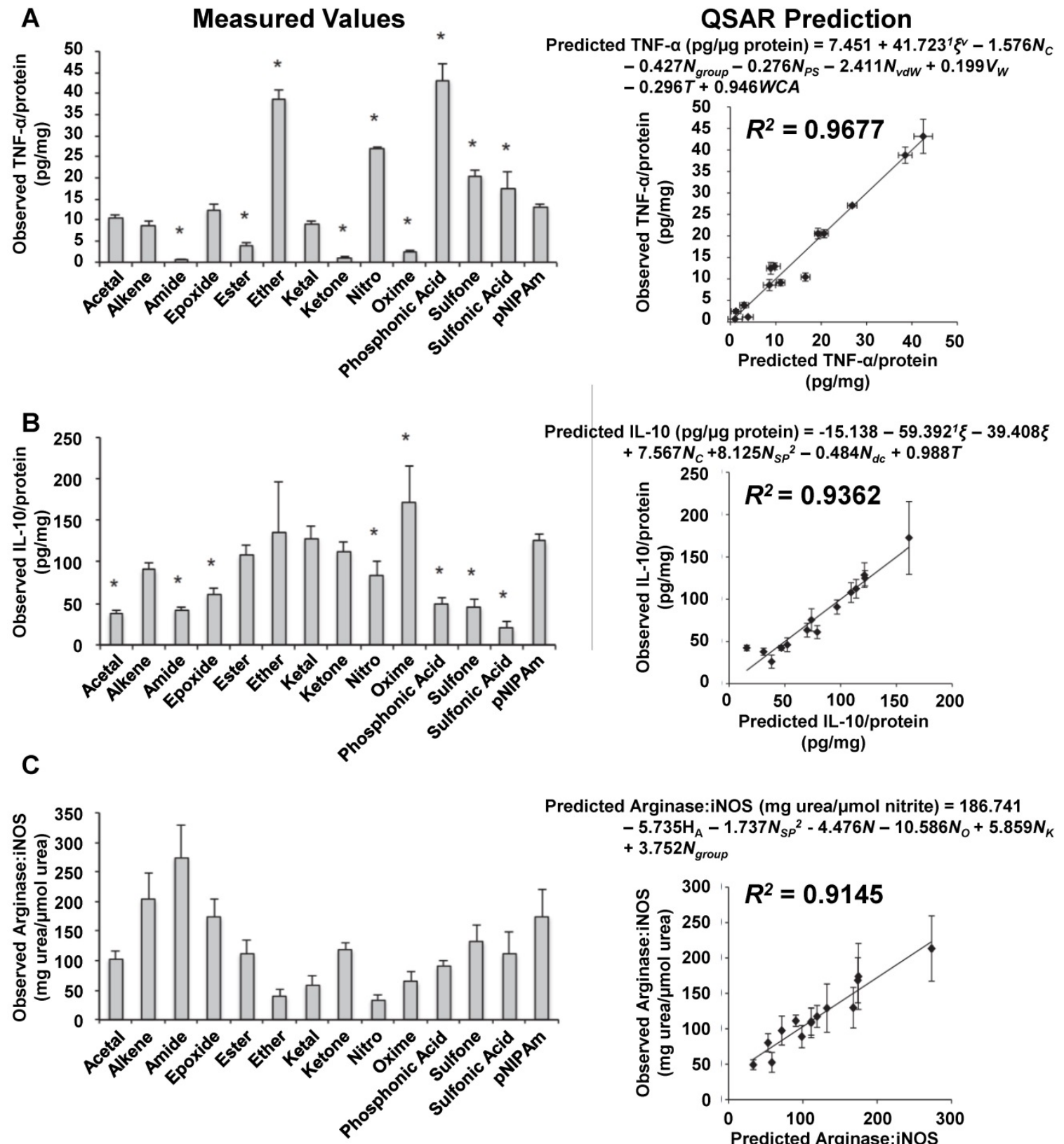

Predicted Arginase:iNOS (mg urea/ $\mu \mathrm{mol}$ nitrite) $=186.741$ $-5.735 \mathrm{H}_{\mathrm{A}}-1.737 \mathrm{~N}_{S P^{2}}-4.476 \mathrm{~N}-10.586 \mathrm{~N}_{\mathrm{O}}+5.859 \mathrm{~N}_{K}$ $+3.752 N_{\text {group }}$

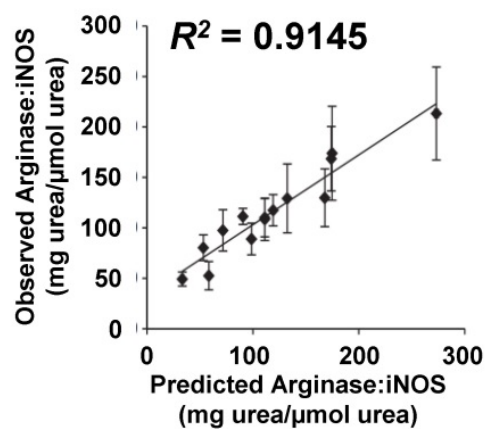

Figure 4. Development of qualitative structure-activity relationships to describe macrophage polarization following treatment with various polymeric nanoparticles. Mice were treated with various nanoparticles, and their macrophages were isolated and characterized. TNF- $\alpha$ secretion was measured from each group of isolated macrophages (A). IL-10 secretion was measured (B). The ratio of arginase to iNOS activity was measured (C). Mathematical relationships were developed using these observed values to predict these values based on nanoparticles properties. To verify the accuracy of these predictions, the observed values were compared to the predicted values for each nanoparticle treatment. Figure was adapted with permission from [80]. 
In contrast to the previously described nanoparticles that are formed by charge neutralization of cationic polymers, unimolecular polymeric nanoparticles containing cationic groups in the core have also been developed. These nanoparticles are expected to remain stable upon dilution and shield charged groups from the nanoparticle surface. Bernal et al. prepared unimolecular nanoparticles by conjugating mannose to the surface of PEI [92]. Mannose-conjugated PEI (Man-PEI) treatments slightly decreased IL-6 mRNA expression increases in response to LPS pre-treatment, but cytokine secretion from macrophages in response to LPS treatment was mostly unaffected by Man-PEI [93]. Man-PEI treatments mitigated LPS-induced increases in IL-6 and IL-10 secretion from macrophages during a second LPS stimulation. These results suggest that while cationic polymer treatments typically cause M1-like polarization, chemical modifications to these polymers to prevent nanoparticle dissociation and charge-based interactions can mitigate their macrophage polarization capabilities.

\section{Liposomes}

Liposomal formulations of anticancer drugs such as FDA-approved Doxil (doxorubicin) or Onivyde (irinotecan) have reduced chemotherapy drug toxicity compared to traditional formulations [94, 95], but the effects of liposome treatments on macrophage polarization have only recently been considered. Towards this goal, Rajan et al. treated tumor-bearing mice with PEG-coated liposome nanoparticles (PLNs) and measured their effect on tumor growth [96]. Figure 5 summarizes these findings and presents a mechanism for PLN effects on tumor growth. PLN treatment increased tumor growth in normal mice compared to saline treatment; however, PLN treatment did not affect tumor growth in macrophage-depleted mice (Figure 5A). To further investigate the effects of PLN treatments on TAMs, the group prepared PLNs that were loaded with alendronate, a bisphosphonate that has been implicated to interfere with TAM activity [97]. The treatment of mice with alendronate-loaded PLN (PLN-ALEN) reduced tumor growth compared to either empty liposome treatments or a physical mixture of empty liposomes and alendronate (Figure 5B). In addition, tumor-bearing mice treated with PLN-ALEN had longer overall survival than PLN-treated mice (Figure 5C). Based on these findings and supplemental biological characterization of cells isolated from liposome-treated tumors, the group proposed a mechanism of macrophage polarization by liposome treatment involving an autocrine/paracrine feedback loop of TGF- $\beta$ that increases CCL-2 secretion, which recruits other myeloid-derived cells to tumors that further enhance the immunosuppressive properties of the tumor microenvironment (Figure 5D). Together, these cells are believed to suppress the activity of cytotoxic $\mathrm{T}$ lymphocytes within tumors and increase tumor survival and progression. The results of this group highlight the complex relationship between nanoparticle treatments, immune cells and macrophage polarization within tumors.

The macrophage polarization effects of nanoparticles such as liposomes can be difficult to measure in vitro, and contradictory conclusions about nanoparticle properties are often reported in the literature. Although differences in liposome chemical composition between studies may contribute to these contradictory conclusions, experimental design differences are also likely to contribute. To assure that the observed macrophage polarization properties of a nanoparticle are accurate and representative of macrophage behavior in the tumor microenvironment, great care must be taken when designing experiments to observe nanoparticle-induced macrophage polarization. In agreement with Rajan et al., other groups observed M2-like polarization in macrophages isolated from dipalmitoyl phosphatidylcholine (DPPC)/cholesterol liposome-treated mice [98]. However, Bartneck et al. reported that PEG-coated liposome treatments did not affect protein expression or cytokine secretion from macrophages in vitro [99]. This paradoxical observation suggests that TAM polarization requires interactions between cancer cells and tumor-associated macrophages to occur, and that in vitro experiments must be carefully designed to mimic these in vivo conditions and observe relevant macrophage behavior to the tumor microenvironment.

\section{Carbon-Based Nanoparticles}

Carbon-based nanoparticles are composed primarily of carbon nanotubes (CNTs) and carbon nanospheres (CNSs), which are also called buckyballs. These nanostructures have unique mechanical properties, high conductivity and the capability to encapsulate both drugs and imaging agents [100-102]. Because of these properties, these materials have been extensively studied and used as reinforcements for metallic implants and to deliver drugs. For these reasons, understanding the harmful effects of carbon-based nanoparticles on macrophages is important. In contrast to other types of nanoparticles, Smith et al. demonstrated that following intravenous injection of CNTs, a significant portion of CNTs were 
localized within circulating Ly-6Chi monocytes [103]. The number of CNT-loaded monocytes entering tumors increased up to 7 days post-treatment, and labelling the CNTs with RGD peptide increased the fraction of monocyte-entrapped CNTs within tumors. However, it should be noted that more than 70 percent of CNTs remained outside of these monocytes. Nonetheless, these results suggest that the unique cellular distribution of CNTs may reduce their localization within macrophages following intravenous administration and lessen their macrophage polarization potential.

In addition to intravenous administration of CNTs, these nanoparticles have also been injected intracranially. VanHandel et al. compared the effects of CNT treatment on macrophages in both healthy and brain tumor-bearing mice [104]. Following intracranial CNT injections, brain tumors in mice had reduced macrophage recruitment compared to injection sites of healthy mice brains. The macrophages isolated from brain tumors had increased TNF-a, IL-6, IL-12 and IL-10 secretion compared to macrophages isolated from healthy mice brain injection sites. Despite these observed cytokine secretion effects in brain tumors, intracranial CNT injections did not affect brain tumor growth compared to untreated mice, presumably because the macrophage polarization effects were only transient. In different studies, other groups have also observed that CNT treatments induce M1-like polarization of macrophages in the lungs and liver $[105,106]$. Meng et al. also reported that CNT treatment slightly increased cytokine secretion, TLR4 expression and CD206 expression on macrophages [107]. However, it should be noted that many of the effects of CNT treatments on macrophage polarization are time-dependent. Long-term studies of macrophage polarization following CNT treatment indicated that iNOS expression and TNF- $\alpha$ secretion increases for 24 to 72 $\mathrm{h}$ post-treatment and returns to normal levels after 7 days, but arginase expression increases for up to 14 days post-treatment [108]. Other studies reported that CNT-treated macrophages returned to an M2-like phenotype 7 days post-treatment [109]. The time-dependency of nanoparticle-induced macrophage polarization effects has not been thoroughly investigated with other types of nanoparticles. Together, these results emphasize the need to measure the long-term effects of nanoparticles on macrophage polarization.
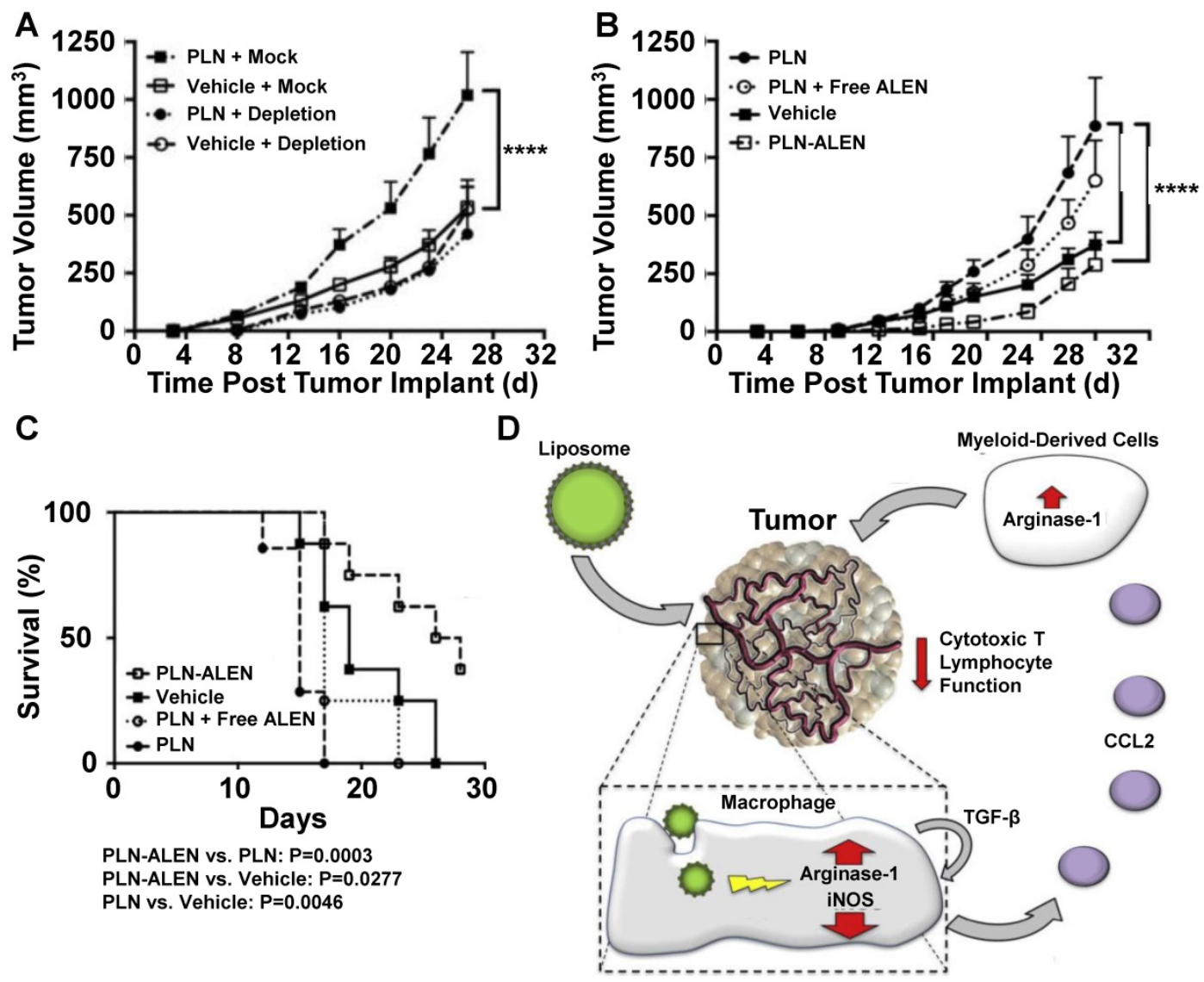

Figure 5. PLN-induced tumor growth is mediated by macrophages. In vivo depletion of systemic macrophages abolished PLN-induced tumor growth (A). PLN-induced tumor progression is mitigated by encapsulated alendronate but not free alendronate (B). Survival of PLN- and PLN-ALEN-treated mice (C). Proposed mechanisms of PLN-induced tumor growth and immunosuppression in tumors based on interactions between liposomes, macrophages and other immune cells ( $\mathbf{D})$. Adapted with permission from [96]. 
The macrophage polarization effects of carbon nanospheres (CNS) have also been investigated. Tang et al. treated macrophages with polyhydroxylated CNS and measured treatment effects on macrophage polarization [110]. CNS-treated macrophages demonstrated a 1.2- to 1.5-fold increase in TNF-a, IL-1 $\beta$ and IL-6 secretion compared to untreated macrophages, indicating a mild M1-like polarization state. In a macrophage-cancer cell co-culture system, CNS-pretreated macrophages decreased the viability of cancer cells compared to non-pretreated macrophages, despite small changes to macrophage cytokine secretion profiles. However, CNS treatments did not affect cancer cells in the absence of macrophages. Based on these results, the group hypothesized that CNS treatments activate macrophages to selectively kill cancer cells. To further investigate this hypothesis, CNS-pretreated macrophages were infused into the peritoneal cavity of mice in a lung metastasis model. The infusion of CNS-pretreated macrophages decreased metastatic nodule formation in mice following an intravenous injection of lung cancer cells compared to mice infused with saline. In contrast, there was no difference in metastatic nodule formation between mice infused with non-pretreated macrophages and mice infused with saline, indicating that CNS-treated macrophages can kill cancer cells in vivo despite moderate changes to their in vitro cytokine secretion profiles. Pacor et al. also found that CNS with a positive surface charge reduced their intracellular accumulation, reduced their mitochondrial localization, and decreased ROS generation in macrophages compared to CNS with an uncharged surface [111]. Taken together, these findings suggest that carbon-based nanoparticles induce short-term M1 macrophage polarization following treatment regardless of their shape, and this polarization can cause cancer cell death in metastatic tumors but may not significantly affect primary tumors.

\section{Biological-Based Nanoparticles}

Nanoparticles consisting of biomolecules, such as proteins, nucleic acids, sugars, lipids and lipoproteins, or cellular components, such as exosomes and microvesicles, have gathered recent interest as biocompatible drug delivery systems. Because some of these nanoparticles have only been developed recently, their macrophage polarization properties have not been thoroughly characterized, and additional studies are needed to elucidate their biological effects. Due to the chemical diversity found within this nanoparticle group, these nanoparticles often have disparate polarization effects. As a result, these nanoparticles were not included in Table 2.
Some examples of biological-based nanoparticles are Abraxane (nanoparticle albumin-bound paclitaxel, or nab-paclitaxel) [112] and the spherical nucleic acid-based nanoparticle NU-0129 is undergoing clinical trials. Cullis et al. investigated the macrophage polarization properties of Abraxane nanoparticles in pancreatic cancer in detail [113]. Abraxane treatment of macrophages increased the in vitro secretion of IL-1 $\alpha$, IL-1 $\beta$, IL-6, IL-12 and TNF- $\alpha$ compared to free paclitaxel treatment, indicating a shift to an M1-like polarization state. Macrophage treatment with a TLR4 inhibitor abrogated the M1-like polarizing effects of Abraxane. These results concur with other reports describing the TLR4-associated effects of paclitaxel on cancer cells and macrophages [114, 115]. To further investigate the in vivo effects of Abraxane in pancreatic cancer treatment, Cullis et al. treated mice bearing orthotopic pancreatic tumors with Abraxane nanoparticles and gemcitabine, which is a clinically used drug combination for pancreatic cancer treatment [116]. Figure 6 describes some of the findings in this experiment. Immunohistochemical staining of tumors demonstrated that the tumors contained F4/80-labelled macrophages (Figure 6A). Fluorescence imaging of tumors that were treated with Oregon green-labelled Abraxane showed that the F4/80-labelled macrophages took up Abraxane (Figure 6B). Abraxane treatment increased the presence of M1-like polarized macrophages, as indicated by MHCII/CD80/CD86 ${ }^{+}$cells (Figure 6C). The increased amount of MHCII/CD80/IL-1 $\alpha^{+}$cells confirmed this M1 polarization observation (Figure 6D). However, gemcitabine treatment alone had no effect on macrophage polarization. This study highlights the potential for drug-loaded albumin-based nanoparticles to amplify the M1-polarizing effects of a drug payload to improve tumor treatment.

Sugar- and lipid-based nanoparticles have been used as biocompatible polymers for drug delivery. Oxidized dextran treatment of macrophages was shown to increase CD206 expression but had no effect on CD86 expression, suggesting that polysaccharide treatments can cause M2-like polarization shifts in macrophages [117]. In addition, oxidized dextran treatments reduced LPS-induced increases in CD86 expression. The treatment of macrophages with lipid-based nanoparticles consisting of phosphatidylserine decreased MCP-1, TNF- $a$, IL-6 mRNA levels and increased TGF- $\beta$ and IL-10 mRNA levels [118]. These results suggest that sugar- and lipid-based nanoparticles can induce weak M2-like polarization in the absence of a drug payload.

Researchers have also investigated the effects of nucleic acid-based nanoparticles on macrophages. 
A
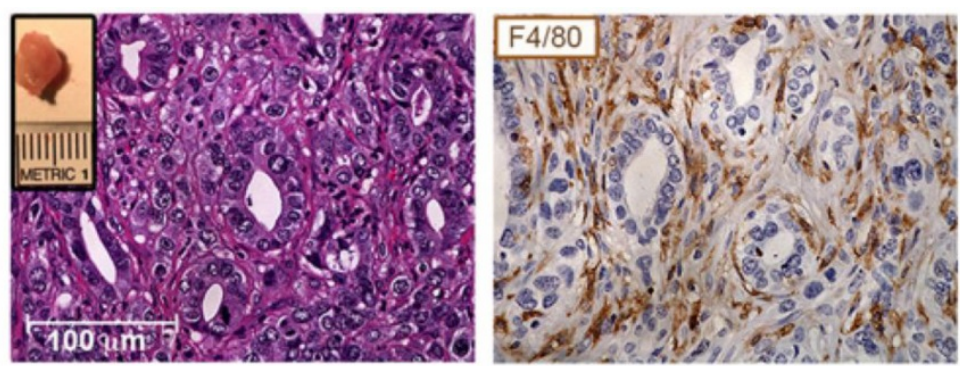

B

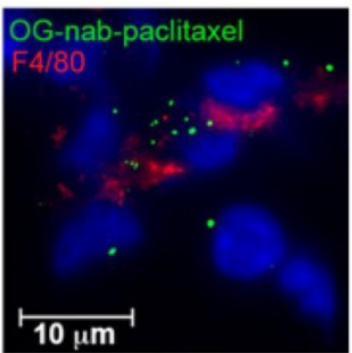

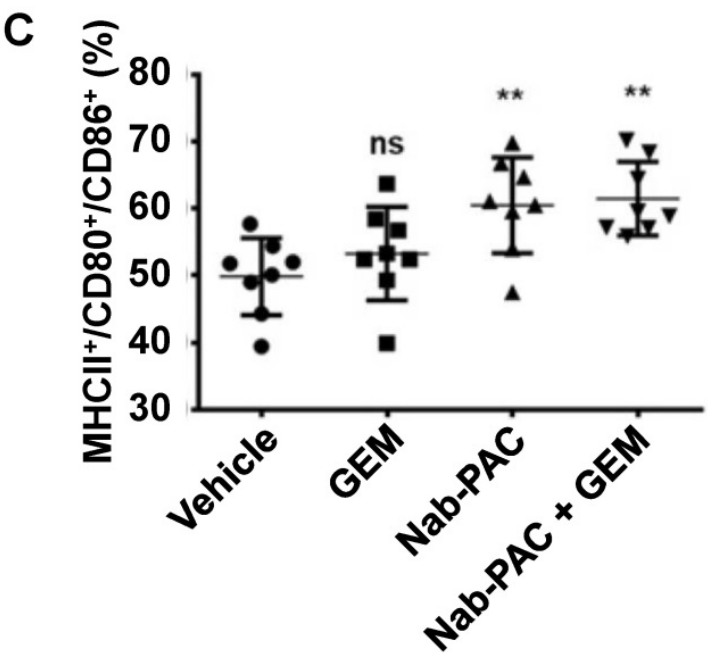

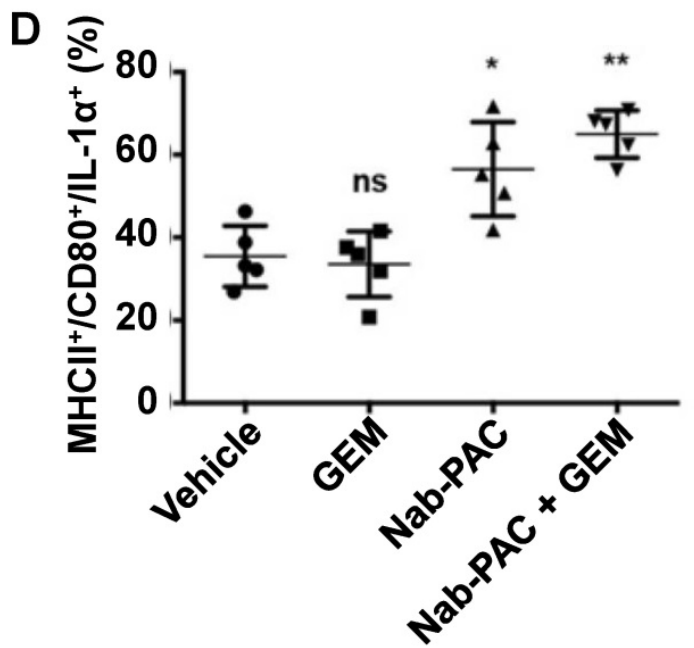

Figure 6. Nab-paclitaxel treatment induces in vivo Ml activation of pancreatic tumor-associated macrophages. Hematoxylin and eosin (H\&E) and $\mathrm{F} 4 / 80$ immunohistochemistry staining of tumors ten days post-implantation indicating macrophage infiltration of tumors (A). The inset shows a representative tumor. Cryo-immunofluorescent analysis of orthotopic tumors resected 2 weeks after implantation and treated with 100 mg Oregon green-labelled nab-paclitaxel ex vivo, followed by immunofluorescent staining with anti-F4/80 (B). Quantification of MHCII/CD80/CD86+ cells in orthotopic tumors 48 hours post-treatment (C). Quantification of MHCII/CD80/IL-1 $\alpha^{+}$cells in orthotopic tumors 48 hours post-treatment (D). *, p<0.05; **, p< 0.01; ns, not significant. Reprinted by permission from the American Association for Cancer Research: Cullis et al., Macropiniocytosis of Nab-paclitaxel Drives Macrophage Activation in Pancreatic Cancer, Cancer Immunology Research, March 2017, 5, 3, 182-190 [113].

Macrophage treatment with tetrahedral DNA nanostructures (TDNs) increased TNF- $\alpha$, IL-1 $\beta$ and IL-6 secretion from macrophages [119]. TDNs slightly increased iNOS expression and decreased arginase expression in macrophages. Zhang et al. also demonstrated that DNA-based nanoflowers increased TNF- $\alpha$, IL-6 and IL-10 secretion from macrophages [120]. In contrast to DNA-based nanoparticles, RNA-based nanoparticle treatments did not affect TNF- a or IL-6 secretion from macrophages [121]. To directly compare the effects of various types of nucleic acid-based nanoparticles on cytokine activation, Afonin et al. prepared DNA-based and RNA-based nanoparticles with similar structures and treated peripheral blood mononuclear cells (PBMCs) [122]. In these studies, RNA nanoparticles significantly increased IL-1 $\beta$ secretion from PBMCs while DNA nanoparticles had no effect. Differences between this study and other studies could be attributed to biological differences between PBMCs and macrophages. Overall, these findings suggest that the structure, conformation and chemical composition of nucleic acid-based nanoparticles can significantly affect their macrophage polarization potential.

\section{Metallic Nanoparticles}

Metallic nanoparticles can enter the body due to either environmental exposure or the breakdown of metallic implants [123-125]. Although the cytotoxicity of these nanoparticles in various cell types has been studied, the macrophage polarization properties of these nanoparticles are less thoroughly described in the literature. Armstead et al. demonstrated that tungsten carbide-cobalt nanoparticle (WC-Co-NP) treatment decreased CD206 expression on macrophages in a co-culture system of macrophages and healthy endothelial cells [126]. WC-Co-NP-treated macrophages also demonstrated increased TNF- $\alpha$ and IL-1 $\beta$ secretion. Scherbart et al. investigated the size-dependent effects of titanium nanoparticles (Ti-NPs) on macrophage polarization [127]. Smaller diameter Ti-NP treatments increased HO-1 expression, iNOS expression and TNF-a secretion from macrophages, while larger diameter Ti-NP treatments had no effect on these three 
polarization markers. However, macrophage treatment with either diameter nanoparticle increased ROS generation in macrophages. Kumar et al. also found that Ti-NP treatments increased IL- $1 \beta$, IL- 6 and TNF- $\alpha$ secretion in macrophages that were isolated from mice [128]. Furthermore, Sarkar et al. demonstrated that macrophages that were treated using silver nanoparticles with various diameters and surface coatings increased IL-1 $\beta$ and IL-8 mRNA expression in cells [129]. Together, these results suggest that various types of ROS-generating metallic nanoparticles can induce M1-like polarization in macrophages.

In contrast to many metallic nanoparticles, cerium oxide nanoparticles (nanoceria) have demonstrated antioxidant properties under physiological conditions. The antioxidant properties of nanoceria have been employed to develop biosensors and to inhibit bacterial growth [130-134]. Selvaraj et al. found that nanoceria treatment of macrophages decreased TNF- $\alpha$, IL-1 $\beta$, IL-1 $\alpha$, MIP-3 $\beta$, MCP-1 and MCP-3 secretion following LPS pretreatment [135]. Nanoceria treatment also reduced iNOS expression in macrophages, which decreased ROS generation and ROS-induced liver damage in nanoceria-treated rats. Both nanoceria and copper nanoparticle treatments have been shown to increase arginase expression in macrophages and shift their activity towards M2-like polarization [136, 137]. Similarly, the treatment of macrophages with chromium oxide nanoparticles did not affect TNF-a, MCP-1 or MIP-1a mRNA expression [138]. Taken together, these results indicate that antioxidative nanoparticles can induce M2-like polarization in macrophages.

To better understand the mechanisms behind macrophage polarization following drug-loaded metallic nanoparticle treatment, the potential synergistic effects between drugs and metallic nanoparticles on macrophage polarization must be considered. One example of a drug-loaded metallic nanoparticle with synergistic effects on macrophage polarization is doxorubicin (DOX)-loaded zinc oxide nanoparticles (ZnO-DOX). Zinc oxide nanoparticle treatments were shown to increase M1 marker cytokine secretion from macrophages [139, 140]; however, some anticancer drugs such as DOX have been reported to induce M2-like macrophage polarization [141-143]. Wang et al. investigated the effects of ZnO-DOX on tumors, and Figure 7 describes their approach and some of the key findings in their study related to macrophages. The group proposed that zinc oxide nanoparticles affect macrophages, cancer cells and cancer stem cells within tumors, and a combination of these effects inhibits cancer progression (Figure 7A). While doxorubicin treatment alone decreased TNF-a secretion from macrophages, zinc oxide nanoparticle treatment slightly increased TNF-a secretion (Figure 7B). In contrast to both treatments, ZnO-DOX significantly increased TNF-a secretion from macrophages. $\mathrm{ZnO}-\mathrm{DOX}$ treatments also increased IL-6 secretion in comparison to individual treatments (Figure 7C). Macrophages treated with $\mathrm{ZnO}-\mathrm{DOX}$ also demonstrated increased CD80, CD86 and MHCII expression in comparison to either DOX or zinc oxide nanoparticles (Figure 7D). These findings demonstrated that nanoparticle encapsulation of anticancer drugs altered its macrophage polarization properties synergistically, and that investigators should consider potential synergistic effects between drugs and nanoparticles when designing drug-loaded nanoparticles for effective cancer treatment.

\section{Iron Oxide Nanoparticles}

Iron oxide nanoparticles are currently used clinically as iron replacement therapies [144]. Several groups have also developed iron oxide-based nanoparticles as theranostic drug delivery systems [145-147]. Because iron exposure regulates the expression of iron transport-related proteins that are correlated to macrophage polarization states [148, 149], researchers have hypothesized that iron oxide nanoparticles would have strong effects on macrophage polarization within the tumor microenvironment. As a result, the effects of iron oxide nanoparticle treatments on macrophage polarization within tumors and the therapeutic consequences of iron oxide nanoparticle-induced macrophage polarization have been investigated extensively.

Super-Paramagnetic Iron Oxide Nanoparticles (SPIONs) are iron oxide nanoparticles consisting of an iron oxide core coated with a biocompatible polymer coating such as dextran or polyethylene glycol. Kodali et al. compared the effects of silica nanoparticle and SPION treatments on lung macrophages by measuring changes in gene expressions following either treatment [150]. Their results showed that SPION treatments changed the expression of 1029 genes, while silica nanoparticle treatments changed the expression of only 67 genes. SPION treatments reduced IL-10 secretion from macrophages more than silica nanoparticle treatments. In addition, SPION treatments increased TNF- $\alpha$ secretion from macrophages in comparison to silica nanoparticle treatments. As a result, researchers often consider iron oxide nanoparticles to have stronger M1-like polarization capabilities than other types of M1-polarizing nanoparticles. 

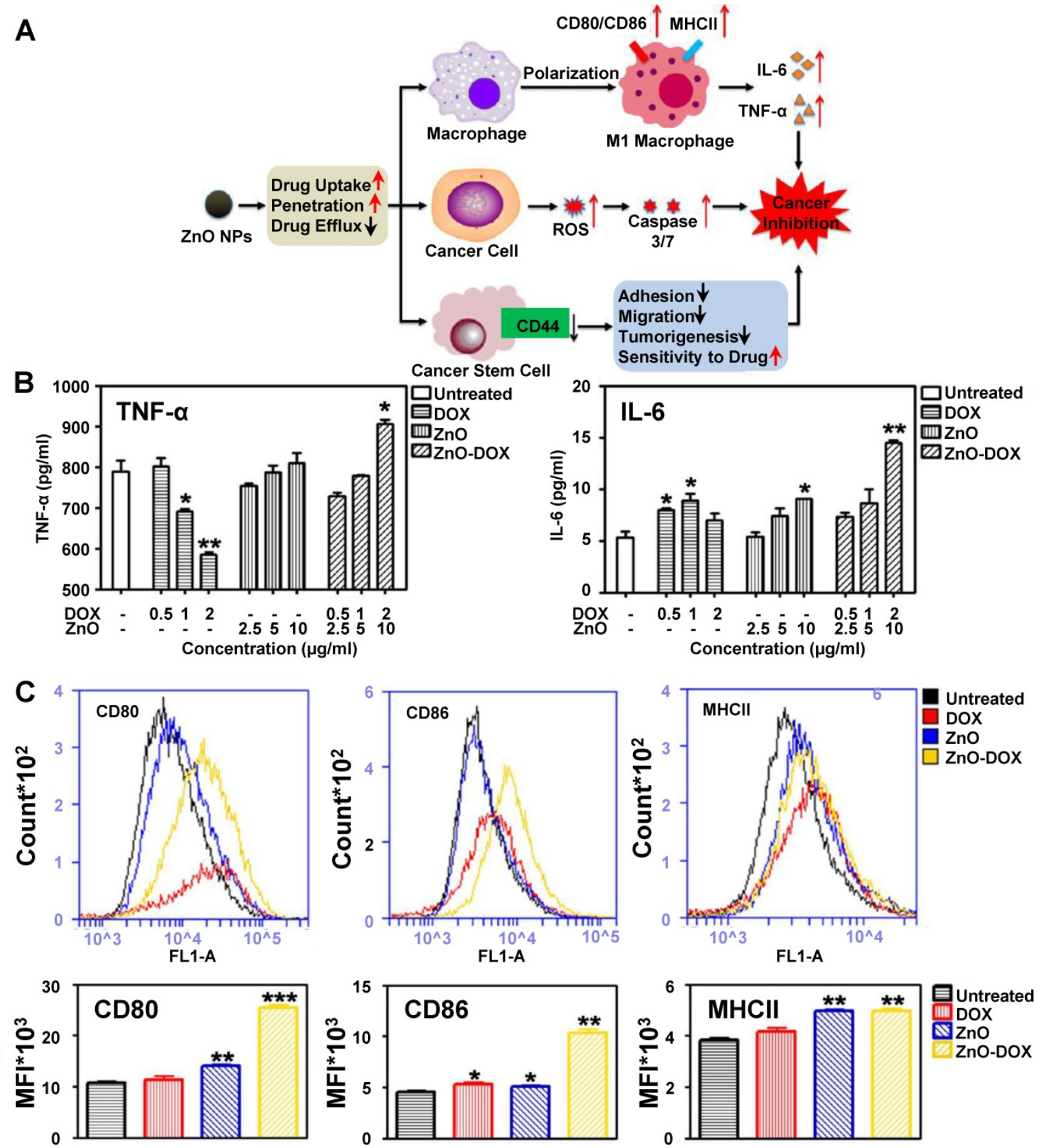

Figure 7. Zinc oxide nanoparticle effects on tumor cells. Proposed mechanism for zinc oxide nanoparticle effects on macrophages, cancer cells and cancer stem cells (A). TNF- $\alpha$ and IL-6 secretion of macrophages treated with either doxorubicin (DOX), zinc oxide nanoparticles ( $\mathrm{ZnO}$ ) or doxorubicin-loaded ZnO (ZnO-DOX) (B). DOX treatment enhances TNF- $\alpha$ and IL-6 secretion increases following ZnO treatment. DOX also enhances CD80 and CD86 increases following $\mathrm{ZnO}$ treatment but has a minimal effect on MHCll expression (C). Adapted with permission from [140]. Copyright 2017 American Chemical Society.

The potential effects of both the surface coating and the iron oxide core on macrophage polarization must be considered. In order to investigate potential surface coating effects on macrophage polarization, Rojas et al. prepared dimercaptosuccinic acid-, aminopropyl silane- and aminodextran-coated SPIONs for macrophage treatment [151]. Macrophage treatment with these SPIONs increased ROS generation and ferroportin expression and shifted macrophages towards an M1-like phenotype regardless of surface coating. The observation of SPION treatment-induced ferroportin expression agrees with previous reports [152, 153]. Other groups demonstrated that macrophage treatment with PEI-coated SPIONs increased CD80, CD86 and ferritin expression on macrophages, while also increasing IL-12 and IL-10 secretion from macrophages [154]. In patients, intravenous injections of amine-functionalized polyvinyl alcohol-coated SPIONs increased IL-1 $\beta$ secretion from isolated macrophages and increased cytokine concentrations in the blood for up to $72 \mathrm{~h}$ [155]. However, Manuelli et al. showed that PEG-coated SPION treatments did not affect TNF-a secretion from macrophages, despite 
observed TNF-a secretion increases from macrophages following citrate-coated and a-Tn mucin antigen-coated SPION treatment [156]. In this study, the macrophage polarization differences between PEG-coated SPION treatments and other SPION treatments were attributed to decreased cellular uptake, which agrees with previous experimental observations of PEG-coated SPION treatments [157, 158]. Taken together, these results suggest that the metallic core of iron oxide nanoparticles, rather than the surface coating, control their macrophage polarization properties.

Feraheme (also called ferumoxytol) is an FDA-approved carboxymethyl dextran-coated iron oxide nanoparticle that is used to treat iron deficient anemia. This nanoparticle has been used as a drug

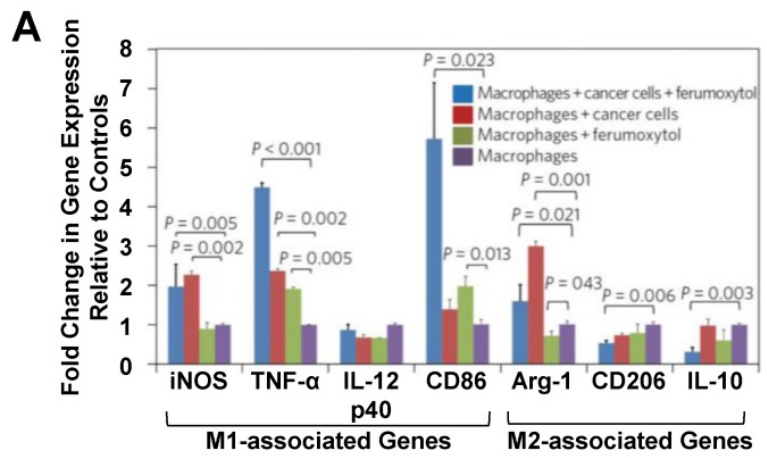

C
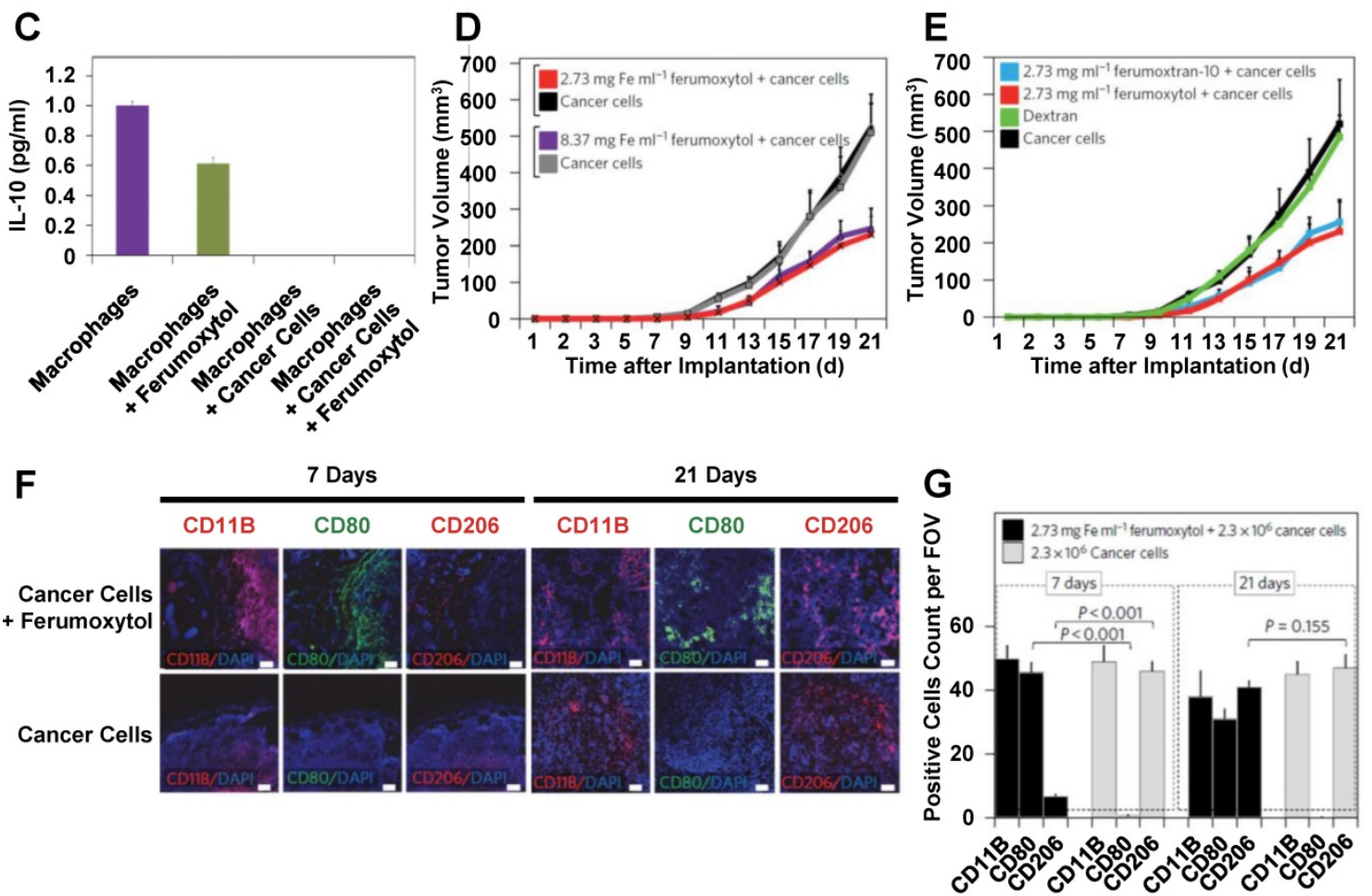

Figure 8. Effects of ferumoxytol treatment on macrophages and tumor growth. Ferumoxytol treatment increased $\mathrm{Ml}$-associated gene expression while also decreasing M2-associated gene expression (A). TNF- $\alpha$ secretion was decreased from ferumoxytol-treated macrophages in the presence of cancer cells (B). Ferumoxytol inhibited IL-10 secretion from macrophages in the presence of cancer cells (C) and also reduced tumor growth in a dose-dependent manner (D). In contrast, dextran nanoparticles had no effect on tumor growth (E). Tissue sections from tumors after 7 or 21 days were stained for CD11B, CD80 or CD206 (F). Ferumoxytol decreased CD206 expression and increased CD80 expression on tumor-associated macrophages for up to 21 days (F). Adapted with permission from [161]. 
injections of dextran did not affect tumor growth, tumor growth effects of ferumoxytol were attributed to the iron oxide core (Figure $8 \mathrm{E}$ ). In addition, tissue sections from ferumoxytol-containing tumors were taken at 7 and 21 days post-injection and analyzed for CD80 and CD206 expression (Figure 8F). Ferumoxytol decreased CD206 expression and increased CD80 expression at 7 days post-injection (Figure 8G). These effects were maintained at 21 days post-injection. These findings suggest that ferumoxytol nanoparticles hold promise to improve cancer treatment by inducing tumor-suppressive macrophage polarization within tumors.

In addition to Feraheme, several other iron oxide-based nanoparticle have been used clinically as MRI contrast agents. The treatment of M2-polarized macrophages with the carboxydextran-coated SPION Resovist (ferucarbotran) increased ferritin, CD86 and cathepsin L expression while also increasing TNF-a secretion, indicating an M1-like polarization shift [162]. However, other reports suggest that macrophage labeling with either the dextran-coated iron oxide nanoparticle Feridex IV (ferumoxide) or the ultra-small SPION Combidex (ferumoxytran-10) had no significant effect on macrophage behavior [163, 164]. The reported differences in macrophage polarization effects between these experiments could be attributed to lower iron doses in macrophage labeling experiments (0.1-0.5 $\mathrm{mg}$ iron/ml media) compared to cancer treatment experiments (3-9 mg iron $/ \mathrm{ml}$ media).

Clinical evidence also indicates that iron accumulation near tumors due to hemolysis induces M1-like polarization. Costa da Silva et al. examined iron accumulations in tissue samples from non-small cell lung cancer patients [165]. In these tissue samples, hemolysis-induced iron accumulation near the periphery of lung tumors was positively correlated to CD68 expression on TAMs and inversely correlated to tumor size in patients. Iron-containing macrophages in tissue samples exhibited increased iNOS and CD86 expression, along with decreased CD206 expression. In addition, these macrophages also demonstrated increased IL-6 secretion and decreased IL-10 secretion, further verifying an M1 polarization shift. This group hypothesized that the observed hemolysis-induced tumor size decreases could also be caused by iron oxide nanoparticle accumulation near tumors. To test this hypothesis, the group treated lung tumor-bearing mice with iron oxide nanoparticles. In these mice, iron accumulation near tumors decreased CD206 expression on TAMs and decreased tumor size. Overall, this research suggests that iron-induced M1-like polarization of TAMs has clinical applications to decrease tumor size and improve cancer therapy.
Alternating magnetic field (AMF) therapy has been proposed as a method for controlling drug release from SPIONs and inducing hyperthermia in tumors [166-168], but AMF effects on macrophage polarization have been less thoroughly studied. Kang et al. investigated the effects of AMF therapy on macrophage polarization following treatment with an RGD peptide-labelled amino-silica-coated SPION [169]. Figure 9 describes their experimental protocol and findings. These SPIONs were attached to a matrix and implanted into mice, which were then exposed to alternating magnetic fields with or without injection of M2-polarizing cytokines (Figure 9A). Following nanoparticle implantation, macrophages in mice that were exposed to a low frequency AMF exhibited decreased iNOS and increased arginase-1 expression in macrophages, indicating an M2-like polarization state (Figure 9B). In contrast, macrophages in mice that were exposed to a high frequency AMF demonstrated an M1-like polarization state. The AMF-induced macrophage polarization effects could also overcome any effects from M1- or M2-polarizing cell media treatment in vitro. Overall, these results suggest that AMF therapy holds promise to control macrophage polarization following nanoparticle treatment.

\section{Conclusions and Future Perspectives}

Nanoparticle-based drug delivery systems offer a unique opportunity to target tumor-associated macrophages, which contribute to tumorigenesis, metastasis and therapeutic resistance in patients. The contributions of nanoparticles to macrophage polarization within the tumor microenvironment must be considered when developing effective nanoparticle-based therapies for cancer treatment. In general, M2-like macrophages increase tumor growth and suppress immune responses to cancer cells, while M1-like macrophages can reduce tumor growth by selectively killing cancer cells within the tumor microenvironment. Because there is no standardized method to measure the effects of a nanoparticle treatment on macrophage polarization, researchers often use inconsistent markers, timepoints and measurement conditions to quantify nanoparticle effects on macrophage polarization. Because of this inconsistency, researchers can report inconsistent findings and conclusions. To overcome this issue, standardized conditions are needed for researchers to measure and report the macrophage polarization effects of nanoparticles. In general, reducing the cellular uptake of a nanoparticle by decreasing its diameter, increasing its surface charge or adding an inert surface coating can reduce its macrophage polarization effects. In contrast, increasing the 

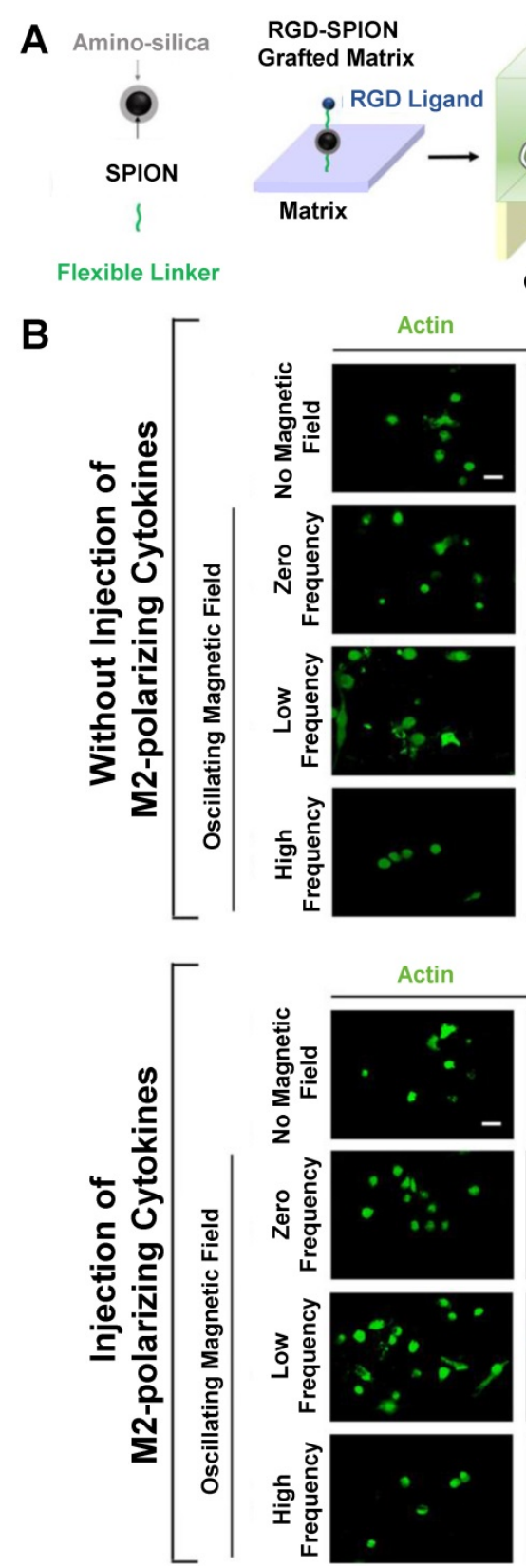
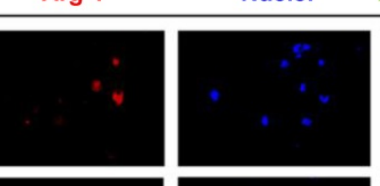

Actin/Arg-1/Nuclei
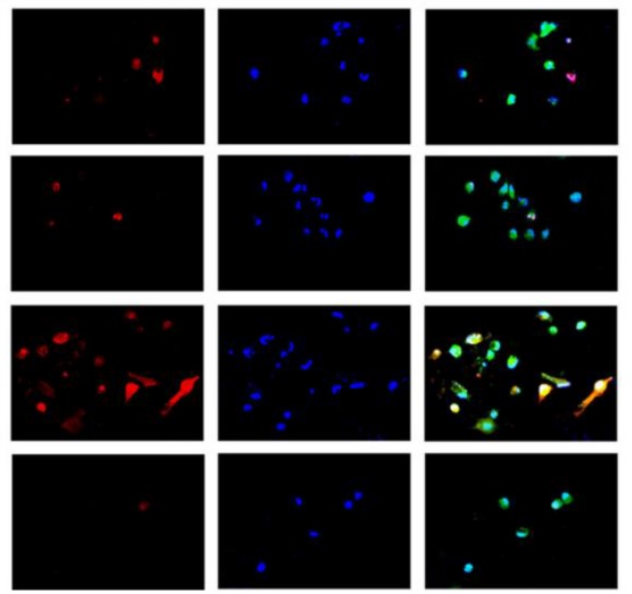

Figure 9. Alternating Magnetic Field (AMF) therapy as an approach to control macrophage polarization. Schematic of AMF-induced macrophage polarization measurement in vivo (A). Low frequency AMF decreased iNOS expression in isolated macrophages, while high frequency AMF increased iNOS expression (B). In contrast, low frequency AMF increased arginase-1 expression in isolated macrophages in M2 polarizing conditions, while high frequency AMF decreased arginase-1 expression. Reproduced with permission from [169]. Copyright 2017 American Chemical Society.

macrophage uptake of a nanoparticle by adding a macrophage-targeting moiety to the surface can enhance its macrophage polarization properties. Macrophage-polarizing drug-loaded nanoparticles may also demonstrate synergistic effects with their therapeutic payload. The literature suggests that polymeric nanoparticles and liposomes can cause M2-like polarization following macrophage treatment, while other types of nanoparticles can cause M1-like polarization. However, a variety of clinically-approved iron oxide nanoparticle treatments have also been shown to induce strong
M1-like polarization in TAMs as compared to other nanoparticles and reduce tumor growth in animal models. In the future, additional work is needed to elucidate both the dose- and time-dependency of nanoparticle treatments on macrophage polarization, particularly for iron oxide nanoparticles. Overall, nanoparticles are a promising treatment approach to modulate macrophage polarization in the tumor microenvironment, and researchers developing nanoparticle-based cancer treatments should consider the macrophage-polarizing potential of nanoparticles to maximize their therapeutic efficacy. 


\section{Abbreviations}

BSA: bovine serum albumin; CD: cluster of differentiation; CNS: carbon nanosphere; CNT: carbon nanotube; Cyto D: cytochalasin D; DOX: doxorubicin; DPPC: dipalmitoyl phosphatidylcholine; ECM: extracellular matrix; FITC: fluorescein isothiocyanate; h: hours; IL: interleukin; LPS: lipopolysaccharide; MHCII: major histocompatibility complex type II; MMP-9: matrix metallopeptidase-9; M 1 : type 1 macrophage; $\mathrm{M} \varphi 2$ : type 2 macrophage; MSN: mesoporous silica nanoparticle; $\mu \mathrm{m}$ : micron; NK cell: natural killer cell; nm: nanometer; PEG: polyethylene glycol; PEI: polyethyleneimine; PGF: placental growth factor; PLGA: poly(lactic-co-glycolic acid); PLN: PEG-coated liposome nanoparticles; PLN-ALEN: Alendronate-loaded PEG-coated liposome nanoparticles; QSAR: quantitative structure-activity relationship; ROS: reactive oxygen species; SPION: superparamagnetic iron oxide nanoparticle; TAM: tumor-associated macrophage; TAT: trans-activating transcriptional activator; TLR: toll-like receptor; VEGF: vascular endothelial growth factor; $\mathrm{ZnO}$ : zinc oxide nanoparticle; ZnO-DOX: doxorubicin-loaded zinc oxide nanoparticle.

\section{Acknowledgements}

The authors thank the National Institute of Health (NIH) for financial support through grant EB 019288 and Cedars Sinai Medical Center for providing additional financial support.

\section{Competing Interests}

The authors have declared that no competing interest exists.

\section{References}

1. Robinson BD, Sica GL, Liu Y-F, Rohan TE, Gertler FB, Condeelis JS, et al. Tumor Microenvironment of Metastasis in Human Breast Carcinoma: A Potential Prognostic Marker Linked to Hematogenous Dissemination. Clinical Cancer Research. 2009; 15: 2433-41.

2. Sun Y, Campisi J, Higano C, Beer TM, Porter P, Coleman I, et al. Treatment-induced damage to the tumor microenvironment promotes prostate cancer therapy resistance through WNT16B. Nature Medicine. 2012; 18. 1359 .

3. Principe DR, DeCant B, Mascarinas E, Wayne EA, Diaz AM, Akagi N, et al. TGFbeta Signaling in the Pancreatic Tumor Microenvironment Promotes Fibrosis and Immune Evasion to Facilitate Tumorigenesis. Cancer Res. 2016; 76: 2525-39.

4. Ma X, Aoki T, Tsuruyama T, Narumiya S. Definition of Prostaglandin $\mathrm{E}<\mathrm{sub}>2</$ sub $>-E P 2$ Signals in the Colon Tumor Microenvironment That Amplify Inflammation and Tumor Growth. Cancer Research. 2015; 75: 2822-32.

5. Allinen M, Beroukhim R, Cai L, Brennan C, Lahti-Domenici J, Huang H, et al. Molecular characterization of the tumor microenvironment in breast cancer. Cancer Cell. 2004; 6: 17-32

6. Ruffell B, Affara NI, Coussens LM. Differential macrophage programming in the tumor microenvironment. Trends in Immunology. 2012 33. 119-26.

7. Cortez-Retamozo V, Etzrodt M, Newton A, Rauch PJ, Chudnovskiy A, Berger $\mathrm{C}$, et al. Origins of tumor-associated macrophages and neutrophils. Proceedings of the National Academy of Sciences. 2012; 109: 2491-6.

8. Franklin RA, Liao W, Sarkar A, Kim MV, Bivona MR, Liu K, et al. The Cellular and Molecular Origin of Tumor-Associated Macrophages. Science. 2014
9. Leek $\mathrm{RD}$, Lewis $\mathrm{CE}$, Whitehouse $\mathrm{R}$, Greenall $\mathrm{M}$, Clarke J, Harris AL Association of macrophage infiltration with angiogenesis and prognosis in invasive breast carcinoma. Cancer Res. 1996; 56: 4625-9.

10. Lin L, Chen Y-S, Yao Y-D, Chen J-Q, Chen J-N, Huang S-Y, et al. CCL18 from tumor-associated macrophages promotes angiogenesis in breast cancer. Oncotarget. 2015; 6: 34758-73.

11. Chanmee T, Ontong P, Konno K, Itano N. Tumor-Associated Macrophages as Major Players in the Tumor Microenvironment. Cancers. 2014; 6: 1670

12. Afik R, Zigmond E, Vugman M, Klepfish M, Shimshoni E, Pasmanik-Chor M, et al. Tumor macrophages are pivotal constructors of tumor collagenous matrix. J Exp Med. 2016; 213: 2315-31.

13. Hashimoto O, Yoshida M, Koma Y, Yanai T, Hasegawa D, Kosaka Y, et al. Collaboration of cancer-associated fibroblasts and tumour-associated macrophages for neuroblastoma development. J Pathol. 2016; 240: 211-23.

14. Ruhland MK, Loza AJ, Capietto A-H, Luo X, Knolhoff BL, Flanagan KC, et al. Stromal senescence establishes an immunosuppressive microenvironment that drives tumorigenesis. Nature Communications. 2016; 7: 11762.

15. Sainz B, Jr., Carron E, Vallespinos M, Machado HL. Cancer Stem Cells and Macrophages: Implications in Tumor Biology and Therapeutic Strategies. Mediators Inflamm. 2016; 2016: 9012369.

16. Vitale M, Cantoni C, Pietra G, Mingari MC, Moretta L. Effect of tumor cells and tumor microenvironment on NK-cell function. Eur J Immunol. 2014; 44: 1582-92.

17. Movahedi K, Laoui D, Gysemans C, Baeten M, Stangé G, Van den Bossche J, et al. Different Tumor Microenvironments Contain Functionally Distinct Subsets of Macrophages Derived from Ly6C(high) Monocytes. Cancer Research. 2010; 70: 5728-39.

18. Hanahan D, Coussens Lisa M. Accessories to the Crime: Functions of Cells Recruited to the Tumor Microenvironment. Cancer Cell. 2012; 21: 309-22.

19. Shapouri-Moghaddam A, Mohammadian S, Vazini H, Taghadosi M, Esmaeili SA, Mardani F, et al. Macrophage plasticity, polarization, and function in health and disease. J Cell Physiol. 2018.

20. Classen A, Lloberas J, Celada A. Macrophage Activation: Classical Vs. Alternative. In: Reiner NE, editor. Macrophages and Dendritic Cells: Methods and Protocols. Totowa, NJ: Humana Press; 2009. p. 29-43.

21. Han MS, Jung DY, Morel C, Lakhani SA, Kim JK, Flavell RA, et al. JNK Expression by Macrophages Promotes Obesity-Induced Insulin Resistance and Inflammation. Science. 2013; 339: 218-22.

22. Lumeng CN, Bodzin JL, Saltiel AR. Obesity induces a phenotypic switch in adipose tissue macrophage polarization. The Journal of Clinical Investigation. 2007; 117: 175-84.

23. Brahmer JR, Lacchetti C, Schneider BJ, Atkins MB, Brassil KJ, Caterino JM, et al. Management of Immune-Related Adverse Events in Patients Treated With Immune Checkpoint Inhibitor Therapy: American Society of Clinical Oncology Clinical Practice Guideline. Journal of Clinical Oncology. 2018; 36: 1714-68

24. Rath M, Muller I, Kropf P, Closs EI, Munder M. Metabolism via Arginase or Nitric Oxide Synthase: Two Competing Arginine Pathways in Macrophages. Frontiers in immunology. 2014; 5: 532

25. Murray Peter J, Allen Judith E, Biswas Subhra K, Fisher Edward A, Gilroy Derek W, Goerdt S, et al. Macrophage Activation and Polarization: Nomenclature and Experimental Guidelines. Immunity. 2014; 41: 14-20.

26. Sica A, Erreni M, Allavena P, Porta C. Macrophage polarization in pathology. Cellular and Molecular Life Sciences. 2015; 72: 4111-26.

27. Liu C-Y, Xu J-Y, Shi X-Y, Huang W, Ruan T-Y, Xie P, et al. M2-polarized tumor-associated macrophages promoted epithelial-mesenchymal transition in pancreatic cancer cells, partially through TLR4/IL-10 signaling pathway. Laboratory Investigation. 2013; 93: 844.

28. Colegio OR, Chu N-Q, Szabo AL, Chu T, Rhebergen AM, Jairam V, et al. Functional polarization of tumour-associated macrophages by tumour-derived lactic acid. Nature. 2014; 513: 559.

29. Goswami KK, Ghosh T, Ghosh S, Sarkar M, Bose A, Baral R. Tumor promoting role of anti-tumor macrophages in tumor microenvironment. Cell Immunol. 2017; 316: 1-10.

30. Sousa S, Brion R, Lintunen M, Kronqvist P, Sandholm J, Mönkkönen J, et al. Human breast cancer cells educate macrophages toward the M2 activation status. Breast Cancer Research. 2015; 17: 101

31. Georgoudaki AM, Prokopec KE, Boura VF, Hellqvist E, Sohn S, Ostling J, et al. Reprogramming Tumor-Associated Macrophages by Antibody Targeting Inhibits Cancer Progression and Metastasis. Cell Rep. 2016; 15: 2000-11.

32. Song M, Liu T, Shi C, Zhang X, Chen X. Bioconjugated Manganese Dioxide Nanoparticles Enhance Chemotherapy Response by Priming Tumor-Associated Macrophages toward M1-like Phenotype and Attenuating Tumor Hypoxia. ACS Nano. 2016; 10: 633-47

33. Han J, Zhen J, Du Nguyen V, Go G, Choi Y, Ko SY, et al. Hybrid-Actuating Macrophage-Based Microrobots for Active Cancer Therapy. Scientific reports. 2016; 6: 28717

34. Santra S, Kaittanis C, Grimm J, Perez JM. Drug/dye-loaded, multifunctional iron oxide nanoparticles for combined targeted cancer therapy and dual optical/magnetic resonance imaging. Small. 2009; 5: 1862-8.

35. Kaittanis C, Shaffer TM, Ogirala A, Santra S, Perez JM, Chiosis G, et al. Environment-responsive nanophores for therapy and treatment monitoring via molecular MRI quenching. Nature Communications. 2014 $5 \cdot 3384$

36. Hobbs SK, Monsky WL, Yuan F, Roberts WG, Griffith L, Torchilin VP, et al. Regulation of transport pathways in tumor vessels: Role of tumor type and 
microenvironment. Proceedings of the National Academy of Sciences. 1998; 95: 4607-12.

37. Stylianopoulos T, Soteriou K, Fukumura D, Jain RK. Cationic Nanoparticles Have Superior Transvascular Flux into Solid Tumors: Insights from a Mathematical Model. Annals of Biomedical Engineering. 2013; 41: 68-77.

38. Chauhan VP, Stylianopoulos T, Martin JD, Popović Z, Chen O, Kamoun WS, et al. Normalization of tumour blood vessels improves the delivery of nanomedicines in a size-dependent manner. Nature nanotechnology. 2012; 7: 383.

39. Hirayama D, Iida T, Nakase H. The Phagocytic Function of Macrophage-Enforcing Innate Immunity and Tissue Homeostasis. Int J Mol Sci. 2017; 19 .

40. Yang B, Han X, Ji B, Lu R. Competition Between Tumor and Mononuclear Phagocyte System Causing the Low Tumor Distribution of Nanoparticles and Strategies to Improve Tumor Accumulation. Curr Drug Deliv. 2016; 13: 1261-74.

41. Soma CE, Dubernet C, Barratt G, Benita S, Couvreur P. Investigation of the role of macrophages on the cytotoxicity of doxorubicin and doxorubicin-loaded nanoparticles on M5076 cells in vitro. J Control Release. 2000; 68: 283-9.

42. Miller MA, Zheng YR, Gadde S, Pfirschke C, Zope H, Engblom C, et al. Tumour-associated macrophages act as a slow-release reservoir of nano-therapeutic Pt(IV) pro-drug. Nat Commun. 2015; 6: 8692.

43. Jackute J, Zemaitis M, Pranys D, Sitkauskiene B, Miliauskas S, Vaitkiene S, et al. Distribution of M1 and M2 macrophages in tumor islets and stroma in relation to prognosis of non-small cell lung cancer. BMC Immunology. 2018; 19:3.

44. Yang M, Li Z, Ren M, Li S, Zhang L, Zhang X, et al. Stromal Infiltration of Tumor-Associated Macrophages Conferring Poor Prognosis of Patients with Basal-Like Breast Carcinoma. J Cancer. 2018; 9: 2308-16.

45. Kawachi A, Yoshida H, Kitano S, Ino Y, Kato T, Hiraoka N. Tumor-associated CD204(+) M2 macrophages are unfavorable prognostic indicators in uterine cervical adenocarcinoma. Cancer Sci. 2018; 109: 863-70.

46. Krausgruber T, Blazek K, Smallie T, Alzabin S, Lockstone H, Sahgal N, et al. IRF5 promotes inflammatory macrophage polarization and TH1-TH17 responses. Nature Immunology. 2011; 12: 231.

47. Barros MHM, Hauck F, Dreyer JH, Kempkes B, Niedobitek G. Macrophage Polarisation: an Immunohistochemical Approach for Identifying M1 and M2 Macrophages. PloS one. 2013; 8: e80908.

48. Vogel DYS, Glim JE, Stavenuiter AWD, Breur M, Heijnen P, Amor S, et al. Human macrophage polarization in vitro: Maturation and activation methods compared. Immunobiology. 2014; 219: 695-703.

49. Lisi L, Ciotti GMP, Braun D, Kalinin S, Currò D, Dello Russo C, et al. Expression of iNOS, CD163 and ARG-1 taken as M1 and M2 markers of microglial polarization in human glioblastoma and the surrounding normal parenchyma. Neuroscience Letters. 2017; 645: 106-12.

50. Srivastava M, Saqib U, Naim A, Roy A, Liu D, Bhatnagar D, et al. The TLR4-NOS1-AP1 signaling axis regulates macrophage polarization. Inflamm Res. 2017; 66: 323-34.

51. Walkey CD, Olsen JB, Guo H, Emili A, Chan WCW. Nanoparticle Size and Surface Chemistry Determine Serum Protein Adsorption and Macrophage Uptake. Journal of the American Chemical Society. 2012; 134: 2139-47.

52. Mantovani A, Polentarutti N, Luini W, Peri G, Spreafico F. Role of host defense mechanisms in the antitumor activity of adriamycin and daunomycin in mice. Journal of the National Cancer Institute. 1979; 63: 61-6.

53. Bruchard $M$, Mignot $G$, Derangère $V$, Chalmin F, Chevriaux A, Végran F, et al. Chemotherapy-triggered cathepsin B release in myeloid-derived suppressor cells activates the Nlrp3 inflammasome and promotes tumor growth. Nature medicine. 2013; 19: 57 .

54. Kodumudi KN, Woan K, Gilvary DL, Sahakian E, Wei S, Djeu JY. A novel chemoimmunomodulating property of docetaxel: suppression of myeloid-derived suppressor cells in tumor bearers. Clinical cancer research. 2010: 1078-0432. CCR-10-733.

55. Shaffer TM, Wall MA, Harmsen S, Longo VA, Drain CM, Kircher MF, et al. Silica Nanoparticles as Substrates for Chelator-free Labeling of Oxophilic Radioisotopes. Nano Letters. 2015; 15: 864-8.

56. Shaffer TM, Harmsen S, Khwaja E, Kircher MF, Drain CM, Grimm J. Stable Radiolabeling of Sulfur-Functionalized Silica Nanoparticles with Copper-64. Nano Letters. 2016; 16: 5601-4.

57. Bradbury MS, Phillips E, Montero PH, Cheal SM, Stambuk H, Durack JC, et al. Clinically-translated silica nanoparticles as dual-modality cancer-targeted probes for image-guided surgery and interventions. Integr Biol (Camb). 2013; 5: 74-86.

58. Kharlamov AN, Tyurnina AE, Veselova VS, Kovtun OP, Shur VY, Gabinsky JL. Silica-gold nanoparticles for atheroprotective management of plaques: results of the NANOM-FIM trial. Nanoscale. 2015; 7: 8003-15.

59. Kwon D, Cha BG, Cho Y, Min J, Park E-B, Kang S-J, et al. Extra-Large Pore Mesoporous Silica Nanoparticles for Directing in Vivo M2 Macrophage Polarization by Delivering IL-4. Nano Letters. 2017; 17: 2747-56.

60. Bancos S, Stevens DL, Tyner KM. Effect of silica and gold nanoparticles on macrophage proliferation, activation markers, cytokine production, and phagocytosis in vitro. International Journal of Nanomedicine. 2015; 10: 183-206.
61. Cejudo-Guillén M, Ramiro-Gutiérrez ML, Labrador-Garrido A, Díaz-Cuenca A, Pozo D. Nanoporous silica microparticle interaction with toll-like receptor agonists in macrophages. Acta Biomaterialia. 2012; 8: 4295-303.

62. Park EJ, Park K. Oxidative stress and pro-inflammatory responses induced by silica nanoparticles in vivo and in vitro. Toxicol Lett. 2009; 184: 18-25.

63. Kusaka T, Nakayama M, Nakamura K, Ishimiya M, Furusawa E, Ogasawara $\mathrm{K}$. Effect of silica particle size on macrophage inflammatory responses. PloS one. 2014; 9: e92634

64. Wan S, Kelly PM, Mahon E, Stöckmann H, Rudd PM, Caruso F, et al. The "Sweet" Side of the Protein Corona: Effects of Glycosylation on Nanoparticle-Cell Interactions. ACS Nano. 2015; 9: 2157-66.

65. Chen YP, Wu SH, Chen IC, Chen CT. Impacts of Cross-Linkers on Biological Effects of Mesoporous Silica Nanoparticles. ACS Applied Materials and Interfaces. 2017; 9: 10254-65.

66. Young KL, Ross MB, Blaber MG, Rycenga M, Jones MR, Zhang C, et al. Using DNA to design plasmonic metamaterials with tunable optical properties. Adv Mater. 2014; 26: 653-9.

67. Chang WS, Wen F, Chakraborty D, Su MN, Zhang Y, Shuang B, et al. Tuning the acoustic frequency of a gold nanodisk through its adhesion layer. Nat Commun. 2015; 6: 7022.

68. Chen W, Ayala-Orozco C, Biswal NC, Perez-Torres C, Bartels M, Bardhan R, et al. Targeting pancreatic cancer with magneto-fluorescent theranostic gold nanoshells. Nanomedicine (Lond). 2014; 9: 1209-22.

69. Harmsen S, Bedics MA, Wall MA, Huang R, Detty MR, Kircher MF. Rational design of a chalcogenopyrylium-based surface-enhanced resonance Raman scattering nanoprobe with attomolar sensitivity. Nature Communications. 2015; 6: 6570 .

70. Zazo H, Colino CI, Warzecha KT, Hoss M, Gbureck U, Trautwein C, et al. Gold Nanocarriers for Macrophage-Targeted Therapy of Human Immunodeficiency Virus. Macromolecular Bioscience. 2017; 17: 1600359.

71. Bastus NG, Sanchez-Tillo E, Pujals S, Farrera C, Lopez C, Giralt E, et al. Homogeneous conjugation of peptides onto gold nanoparticles enhances macrophage response. ACS Nano. 2009; 3: 1335-44.

72. Bastus NG, Sanchez-Tillo E, Pujals S, Farrera C, Kogan MJ, Giralt E, et al. Peptides conjugated to gold nanoparticles induce macrophage activation. Molecular immunology. 2009; 46: 743-8.

73. Pal R, Chakraborty B, Nath A, Singh LM, Ali M, Rahman DS, et al. Noble metal nanoparticle-induced oxidative stress modulates tumor associated macrophages (TAMs) from an M2 to M1 phenotype: An in vitro approach. International Immunopharmacology. 2016; 38: 332-41.

74. Lee S-W, Yun M-H, Jeong SW, In C-H, Kim J-Y, Seo M-H, et al. Development of docetaxel-loaded intravenous formulation, Nanoxel-PM ${ }^{\mathrm{TM}}$ using polymer-based delivery system. Journal of Controlled Release. 2011; 155: 262-71.

75. Lee KS, Chung HC, Im SA, Park YH, Kim CS, Kim S-B, et al. Multicenter phase II trial of Genexol-PM, a Cremophor-free, polymeric micelle formulation of paclitaxel, in patients with metastatic breast cancer. Breast Cancer Research and Treatment. 2008; 108: 241-50.

76. Haller JA, Bandello F, Belfort R, Blumenkranz MS, Gillies M, Heier J, et al. Randomized, Sham-Controlled Trial of Dexamethasone Intravitreal Implant in Patients with Macular Edema Due to Retinal Vein Occlusion. Ophthalmology. 2010; 117: 1134-46.e3.

77. Ye L, Zhang Y, Yang B, Zhou X, Li J, Qin Z, et al. Zwitterionic-Modified Starch-Based Stealth Micelles for Prolonging Circulation Time and Reducing Macrophage Response. ACS Applied Materials \& Interfaces. 2016; 8: 4385-98.

78. Su L, Zhang W, Wu X, Zhang Y, Chen X, Liu G, et al. Glycocalyx-Mimicking Nanoparticles for Stimulation and Polarization of Macrophages via Specific Interactions. Small. 2015; 11: 4191-200.

79. Wang D, Bratlie KM. Influence of Polymer Chemistry on Cytokine Secretion from Polarized Macrophages. ACS Biomaterials Science \& Engineering. 2015; 1: 166-74.

80. Bygd HC, Forsmark KD, Bratlie KM. Altering in vivo macrophage responses with modified polymer properties. Biomaterials. 2015; 56: 187-97.

81. Gabrielson NP, Pack DW. Acetylation of Polyethylenimine Enhances Gene Delivery via Weakened Polymer/DNA Interactions. Biomacromolecules. 2006; 7: 2427-35

82. Christie RJ, Miyata K, Matsumoto Y, Nomoto T, Menasco D, Lai TC, et al. Effect of Polymer Structure on Micelles Formed between siRNA and Cationic Block Copolymer Comprising Thiols and Amidines. Biomacromolecules. 2011; 12: 3174-85.

83. Cortez MA, Godbey WT, Fang Y, Payne ME, Cafferty BJ, Kosakowska KA, et al. The Synthesis of Cyclic Poly(ethylene imine) and Exact Linear Analogues: An Evaluation of Gene Delivery Comparing Polymer Architectures. Journal of the American Chemical Society. 2015; 137: 6541-9.

84. Wei X, Shao B, He Z, Ye T, Luo M, Sang Y, et al. Cationic nanocarriers induce cell necrosis through impairment of $\mathrm{Na}(+) / \mathrm{K}(+)$-ATPase and cause subsequent inflammatory response. Cell research. 2015; 25: 237-53.

85. Huang Z, Yang Y, Jiang Y, Shao J, Sun X, Chen J, et al. Anti-tumor immune responses of tumor-associated macrophages via toll-like receptor 4 triggered by cationic polymers. Biomaterials. 2013; 34: 746-55.

86. Lunov O, Syrovets T, Loos C, Nienhaus GU, Mailänder V, Landfester K, et al. Amino-Functionalized Polystyrene Nanoparticles Activate the NLRP3 Inflammasome in Human Macrophages. ACS Nano. 2011; 5: 9648-57.

87. Fuchs A-K, Syrovets T, Haas KA, Loos C, Musyanovych A, Mailänder V, et al. Carboxyl- and amino-functionalized polystyrene nanoparticles differentially 
affect the polarization profile of M1 and M2 macrophage subsets. Biomaterials. 2016; 85: 78-87.

88. Castro F, Pinto ML, Silva AM, Pereira CL, Teixeira GQ, Gomez-Lazaro M, et al. Pro-inflammatory chitosan/poly(gamma-glutamic acid) nanoparticles modulate human antigen-presenting cells phenotype and revert their pro-invasive capacity. Acta Biomaterialia. 2017; 63: 96-109.

89. Gonçalves RM, Pereira ACL, Pereira IO, Oliveira MJ, Barbosa MA. Macrophage response to chitosan/poly-( $\gamma$-glutamic acid) nanoparticles carrying an anti-inflammatory drug. Journal of Materials Science: Materials in Medicine. 2015; 26: 167.

90. Ma L, Shen C-a, Gao L, Li D-w, Shang Y-r, Yin K, et al. Anti-inflammatory activity of chitosan nanoparticles carrying NF-kB/p65 antisense oligonucleotide in RAW264.7 macropghage stimulated by lipopolysaccharide. Colloids and Surfaces B: Biointerfaces. 2016; 142: 297-306.

91. Potuck AN, Weed BL, Leifer CA, Chu CC. Electrostatically self-assembled biodegradable microparticles from pseudoproteins and polysaccharide: fabrication, characterization, and biological properties. Biomacromolecules. 2015; 16: 564-77.

92. Bernal L, Alvarado-Vázquez A, Ferreira DW, Paige CA, Ulecia-Morón C, Hill $B$, et al. Evaluation of a nanotechnology-based approach to induce gene-expression in human THP-1 macrophages under inflammatory conditions. Immunobiology. 2017; 222: 399-408.

93. Alvarado-Vazquez PA, Bernal L, Paige CA, Grosick RL, Moracho Vilrriales C, Ferreira DW, et al. Macrophage-specific nanotechnology-driven CD163 overexpression in human macrophages results in an M2 phenotype under inflammatory conditions. Immunobiology. 2017; 222: 900-12.

94. Alibolandi M, Abnous K, Mohammadi M, Hadizadeh F, Sadeghi F, Taghavi S, et al. Extensive preclinical investigation of polymersomal formulation of doxorubicin versus Doxil-mimic formulation. Journal of Controlled Release. 2017; 264: 228-36.

95. Wang-Gillam A, Li C-P, Bodoky G, Dean A, Shan Y-S, Jameson G, et al. Nanoliposomal irinotecan with fluorouracil and folinic acid in metastatic pancreatic cancer after previous gemcitabine-based therapy (NAPOLI-1): a global, randomised, open-label, phase 3 trial. The Lancet. 2016; 387: 545-57.

96. Rajan R, Sabnani MK, Mavinkurve V, Shmeeda H, Mansouri H, Bonkoungou $\mathrm{S}$, et al. Liposome-induced immunosuppression and tumor growth is mediated by macrophages and mitigated by liposome-encapsulated alendronate. Journal of Controlled Release. 2018; 271: 139-48.

97. Rogers TL, Holen I. Tumour macrophages as potential targets of bisphosphonates. Journal of Translational Medicine. 2011; 9: 177

98. Cruz-Leal Y, Lucatelli Laurindo MF, Osugui L, Luzardo MdC, López-Requena A, Alonso ME, et al. Liposomes of phosphatidylcholine and cholesterol induce an M2-like macrophage phenotype reprogrammable to M1 pattern with the involvement of B-1 cells. Immunobiology. 2014; 219: 403-15.

99. Bartneck M, Peters FM, Warzecha KT, Bienert M, van Bloois L, Trautwein C, et al. Liposomal encapsulation of dexamethasone modulates cytotoxicity, inflammatory cytokine response, and migratory properties of primary human macrophages. Nanomedicine: Nanotechnology, Biology and Medicine. 2014; 10: $1209-20$.

100. Feazell RP, Nakayama-Ratchford N, Dai H, Lippard SJ. Soluble Single-Walled Carbon Nanotubes as Longboat Delivery Systems for Platinum(IV) Anticancer Drug Design. Journal of the American Chemical Society. 2007; 129: 8438-9.

101. Liu Z, Chen K, Davis C, Sherlock S, Cao Q, Chen X, et al. Drug Delivery with Carbon Nanotubes for <em>In vivo</em> Cancer Treatment. Cancer Research. 2008; 68: 6652-60.

102. Shi J, Wang L, Gao J, Liu Y, Zhang J, Ma R, et al. A fullerene-based multi-functional nanoplatform for cancer theranostic applications. Biomaterials. 2014; 35: 5771-84.

103. Smith BR, Ghosn EEB, Rallapalli H, Prescher JA, Larson T, Herzenberg LA, et al. Selective uptake of single-walled carbon nanotubes by circulating monocytes for enhanced tumour delivery. Nature nanotechnology. 2014; 9: 481.

104. VanHandel M, Alizadeh D, Zhang L, Kateb B, Bronikowski M, Manohara H, et al. Selective uptake of multi-walled carbon nanotubes by tumor macrophages in a murine glioma model. Journal of Neuroimmunology. 2009; 208: 3-9.

105. Dong J, Ma Q. Macrophage polarization and activation at the interface of multi-walled carbon nanotube-induced pulmonary inflammation and fibrosis. Nanotoxicology. 2018; 12: 153-68.

106. Principi E, Girardello R, Bruno A, Manni I, Gini E, Pagani A, et al. Systemic distribution of single-walled carbon nanotubes in a novel model: alteration of biochemical parameters, metabolic functions, liver accumulation, and inflammation in vivo. International Journal of Nanomedicine. 2016; 11: 4299-316.

107. Meng J, Li X, Wang C, Guo H, Liu J, Xu H. Carbon nanotubes activate macrophages into a M1/M2 mixed status: recruiting naive macrophages and supporting angiogenesis. ACS Appl Mater Interfaces. 2015; 7: 3180-8.

108. Sanchez VC, Weston P, Yan A, Hurt RH, Kane AB. A 3-dimensional in vitro model of epithelioid granulomas induced by high aspect ratio nanomaterials. Particle and fibre toxicology. 2011; 8: 17 .

109. Wang P, Nie X, Wang Y, Li Y, Ge C, Zhang L, et al. Multiwall Carbon Nanotubes Mediate Macrophage Activation and Promote Pulmonary Fibrosis Through TGF- $\beta$ /Smad Signaling Pathway. Small. 2013; 9: 3799-811.

110. Tang J, Chen Z, Sun B, Dong J, Liu J, Zhou H, et al. Polyhydroxylated fullerenols regulate macrophage for cancer adoptive immunotherapy and greatly inhibit the tumor metastasis. Nanomedicine: Nanotechnology, Biology and Medicine. 2016; 12: 945-54.

111. Pacor S, Grillo A, Dordivec L, Zorzet S, Lucafo M, Da Ros T, et al. Effects of Two Fullerene Derivatives on Monocytes and Macrophages. BioMed Research International. 2015; 2015: 13.

112. Hawkins MJ, Soon-Shiong P, Desai N. Protein nanoparticles as drug carriers in clinical medicine. Advanced Drug Delivery Reviews. 2008; 60: 876-85.

113. Cullis J, Siolas D, Avanzi A, Barui S, Maitra A, Bar-Sagi D. Macropinocytosis of Nab-paclitaxel Drives Macrophage Activation in Pancreatic Cancer. Cancer immunology research. 2017; 5: 182-90.

114. Szajnik M, Szczepanski MJ, Czystowska M, Elishaev E, Mandapathil M, Nowak-Markwitz E, et al. TLR4 signaling induced by lipopolysaccharide or paclitaxel regulates tumor survival and chemoresistance in ovarian cancer. Oncogene. 2009; 28: 4353-63.

115. Wanderley CW, Colon DF, Luiz JPM, Oliveira FF, Viacava PR, Leite CA, et al. Paclitaxel Reduces Tumor Growth by Reprogramming Tumor-Associated Macrophages to an M1 Profile in a TLR4-Dependent Manner. Cancer Res. 2018; 78: 5891-900.

116. Von Hoff DD, Ervin T, Arena FP, Chiorean EG, Infante J, Moore M, et al. Increased Survival in Pancreatic Cancer with nab-Paclitaxel plus Gemcitabine. New England Journal of Medicine. 2013; 369: 1691-703.

117. Chechushkov AV, Kozhin PM, Zaitseva NS, Lemza AE, Men'shchikova EB, Troitskii AV, et al. Oxidized Dextran Enhances Alternative Activation of Macrophages in Mice of Opposite Lines. Bull Exp Biol Med. 2016; 160: 783-6.

118. Wang J, Kang YX, Pan W, Lei W, Feng B, Wang XJ. Enhancement of Anti-Inflammatory Activity of Curcumin Using Phosphatidylserine-Containing Nanoparticles in Cultured Macrophages. Int J Mol Sci. 2016; 17.

119. Zhang $Q$, Lin S, Shi S, Zhang T, Ma Q, Tian T, et al. Anti-inflammatory and Antioxidative Effects of Tetrahedral DNA Nanostructures via the Modulation of Macrophage Responses. ACS Applied Materials \& Interfaces. 2018; 10; 3421-30.

120. Zhang L, Zhu G, Mei L, Wu C, Qiu L, Cui C, et al. Self-Assembled DNA Immunonanoflowers as Multivalent CpG Nanoagents. ACS Appl Mater Interfaces. 2015; 7: 24069-74.

121. Guo S, Li H, Ma M, Fu J, Dong Y, Guo P. Size, Shape, and Sequence-Dependent Immunogenicity of RNA Nanoparticles. Molecular therapy Nucleic acids. 2017; 9: 399-408.

122. Afonin KA, Viard M, Kagiampakis I, Case CL, Dobrovolskaia MA, Hofmann J, et al. Triggering of RNA interference with RNA-RNA, RNA-DNA, and DNA-RNA nanoparticles. ACS Nano. 2015; 9: 251-9.

123. Geiser M, Jeannet N, Fierz M, Burtscher H. Evaluating Adverse Effects of Inhaled Nanoparticles by Realistic In Vitro Technology. Nanomaterials (Basel). 2017; 7.

124. Madl AK, Liong M, Kovochich M, Finley BL, Paustenbach DJ, Oberdorster G. Toxicology of wear particles of cobalt-chromium alloy metal-on-metal hip implants Part I: physicochemical properties in patient and simulator studies. Nanomedicine : nanotechnology, biology, and medicine. 2015; 11: 1201-15.

125. Madl AK, Kovochich M, Liong M, Finley BL, Paustenbach DJ, Oberdorster G. Toxicology of wear particles of cobalt-chromium alloy metal-on-metal hip implants Part II: Importance of physicochemical properties and dose in animal and in vitro studies as a basis for risk assessment. Nanomedicine nanotechnology, biology, and medicine. 2015; 11: 1285-98.

126. Armstead AL, Li B. In vitro inflammatory effects of hard metal (WC-Co) nanoparticle exposure. International Journal of Nanomedicine. 2016; 11: 6195-206.

127. Scherbart AM, Langer J, Bushmelev A, van Berlo D, Haberzettl P, van Schooten FJ, et al. Contrasting macrophage activation by fine and ultrafine titanium dioxide particles is associated with different uptake mechanisms. Particle and fibre toxicology. 2011; 8: 31 .

128. Kumar S, Meena R, Paulraj R. Role of Macrophage (M1 and M2) in Titanium-Dioxide Nanoparticle-Induced Oxidative Stress and Inflammatory Response in Rat. Applied biochemistry and biotechnology. 2016; 180: 1257-75.

129. Sarkar S, Leo BF, Carranza C, Chen S, Rivas-Santiago C, Porter AE, et al. Modulation of Human Macrophage Responses to Mycobacterium tuberculosis by Silver Nanoparticles of Different Size and Surface Modification. PloS one. 2015; 10: e0143077.

130. Wang Q, Perez JM, Webster TJ. Inhibited growth of Pseudomonas aeruginosa by dextran- and polyacrylic acid-coated ceria nanoparticles. Int J Nanomedicine. 2013; 8: 3395-9.

131. Kaittanis C, Santra S, Asati A, Perez JM. A cerium oxide nanoparticle-based device for the detection of chronic inflammation via optical and magnetic resonance imaging. Nanoscale. 2012; 4: 2117-23.

132. Perez JM, Asati A, Nath S, Kaittanis C. Synthesis of Biocompatible Dextran-Coated Nanoceria with pH-Dependent Antioxidant Properties. Small. 2008; 4: 552-6.

133. Asati A, Santra S, Kaittanis C, Nath S, Perez JM. Oxidase-Like Activity of Polymer-Coated Cerium Oxide Nanoparticles. Angewandte Chemie. 2009; 121: 2344-8.

134. Asati A, Kaittanis C, Santra S, Perez JM. pH-Tunable Oxidase-Like Activity of Cerium Oxide Nanoparticles Achieving Sensitive Fluorigenic Detection of Cancer Biomarkers at Neutral pH. Analytical Chemistry. 2011; 83: 2547-53.

135. Selvaraj V, Nepal N, Rogers S, Manne NDPK, Arvapalli R, Rice KM, et al. Inhibition of MAP kinase/NF-kB mediated signaling and attenuation of 
lipopolysaccharide induced severe sepsis by cerium oxide nanoparticles. Biomaterials. 2015; 59: 160-71.

136. Arancibia S, Barrientos A, Torrejon J, Escobar A, Beltran CJ. Copper oxide nanoparticles recruit macrophages and modulate nitric oxide, proinflammatory cytokines and PGE2 production through arginase activation. Nanomedicine (Lond). 2016; 11: 1237-51.

137. Ma JY, Zhao H, Mercer RR, Barger M, Rao M, Meighan T, et al. Cerium oxide nanoparticle-induced pulmonary inflammation and alveolar macrophage functional change in rats. Nanotoxicology. 2011; 5: 312-25.

138. VanOs R, Lildhar LL, Lehoux EA, Beaule PE, Catelas I. In vitro macrophage response to nanometer-size chromium oxide particles. Journal of biomedical materials research Part B, Applied biomaterials. 2014; 102: 149-59.

139. Roy R, Parashar V, Chauhan LKS, Shanker R, Das M, Tripathi A, et al. Mechanism of uptake of $\mathrm{ZnO}$ nanoparticles and inflammatory responses in macrophages require PI3K mediated MAPKs signaling. Toxicology in Vitro. 2014; 28: 457-67.

140. Wang J, Lee JS, Kim D, Zhu L. Exploration of Zinc Oxide Nanoparticles as a Multitarget and Multifunctional Anticancer Nanomedicine. ACS Appl Mater Interfaces. 2017; 9: 39971-84.

141. Belgiovine C, D'Incalci M, Allavena P, Frapolli R. Tumor-associated macrophages and anti-tumor therapies: complex links. Cellular and Molecular Life Sciences. 2016; 73: 2411-24

142. Zitvogel L, Galluzzi L, Smyth Mark J, Kroemer G. Mechanism of Action of Conventional and Targeted Anticancer Therapies: Reinstating Immunosurveillance. Immunity. 2013; 39: 74-88.

143. Shree T, Olson OC, Elie BT, Kester JC, Garfall AL, Simpson K, et al. Macrophages and cathepsin proteases blunt chemotherapeutic response in breast cancer. Genes \& development. 2011; 25: 2465-79.

144. Landry R, Jacobs PM, Davis R, Shenouda M, Bolton WK. Pharmacokinetic study of ferumoxytol: a new iron replacement therapy in normal subjects and hemodialysis patients. American journal of nephrology. 2005; 25: 400-10.

145. Gao N, Bozeman EN, Qian W, Wang L, Chen H, Lipowska M, et al. Tumor Penetrating Theranostic Nanoparticles for Enhancement of Targeted and Image-guided Drug Delivery into Peritoneal Tumors following Intraperitoneal Delivery. Theranostics. 2017; 7: 1689-704.

146. Liu T, Shi S, Liang C, Shen S, Cheng L, Wang C, et al. Iron oxide decorated MoS2 nanosheets with double PEGylation for chelator-free radiolabeling and multimodal imaging guided photothermal therapy. ACS nano. 2015; 9: 950-60.

147. Santra S, Kaittanis C, Grimm J, Perez JM. Drug/dye-loaded, multifunctional iron oxide nanoparticles for combined targeted cancer therapy and dual optical/magnetic resonance imaging. small. 2009; 5: 1862-8.

148. Jung M, Weigert A, Mertens C, Rehwald C, Brune B. Iron Handling in Tumor-Associated Macrophages-Is There a New Role for Lipocalin-2? Frontiers in immunology. 2017; 8: 1171.

149. Corna G, Campana L, Pignatti E, Castiglioni A, Tagliafico E, Bosurgi L, et al. Polarization dictates iron handling by inflammatory and alternatively activated macrophages. Haematologica. 2010; 95: 1814-22.

150. Kodali V, Littke MH, Tilton SC, Teeguarden JG, Shi L, Frevert CW, et al. Dysregulation of macrophage activation profiles by engineered nanoparticles. ACS Nano. 2013; 7: 6997-7010.

151. Rojas JM, Sanz-Ortega L, Mulens-Arias V, Gutiérrez L, Pérez-Yagüe S, Barber DF. Superparamagnetic iron oxide nanoparticle uptake alters M2 macrophage phenotype, iron metabolism, migration and invasion. Nanomedicine: Nanotechnology, Biology and Medicine. 2016; 12: 1127-38.

152. Laskar A, Ghosh M, Khattak SI, Li W Yuan XM Degradation of superparamagnetic iron oxide nanoparticle-induced ferritin by lysosomal cathepsins and related immune response. Nanomedicine (Lond). 2012; 7: 705-17.

153. Gu J, Xu H, Han Y, Dai W, Hao W, Wang C, et al. The internalization pathway, metabolic fate and biological effect of superparamagnetic iron oxide nanoparticles in the macrophage-like RAW264.7 cell. Sci China Life Sci. 2011; 54: 793-805.

154. Mulens-Arias V, Rojas JM, Pérez-Yagüe S, Morales MP, Barber DF. Polyethylenimine-coated SPIONs trigger macrophage activation through TLR-4 signaling and ROS production and modulate podosome dynamics. Biomaterials. 2015; 52: 494-506.

155. Strehl C, Gaber T, Maurizi L, Hahne M, Rauch R, Hoff P, et al. Effects of PVA coated nanoparticles on human immune cells. Int J Nanomedicine. 2015; 10: 3429-45.

156. Manuelli M, Fallarini S, Lombardi G, Sangregorio C, Nativi C, Richichi B. Iron oxide superparamagnetic nanoparticles conjugated with a conformationally blocked alpha-Tn antigen mimetic for macrophage activation. Nanoscale. 2014; 6: 7643-55.

157. Park YC, Smith JB, Pham T, Whitaker RD, Sucato CA, Hamilton JA, et al. Effect of PEG molecular weight on stability, T(2) contrast, cytotoxicity, and cellular uptake of superparamagnetic iron oxide nanoparticles (SPIONs). Colloids Surf B Biointerfaces. 2014; 119: 106-14

158. Maurizi L, Papa AL, Dumont L, Bouyer F, Walker P, Vandroux D, et al. Influence of Surface Charge and Polymer Coating on Internalization and Biodistribution of Polyethylene Glycol-Modified Iron Oxide Nanoparticles. J Biomed Nanotechnol. 2015; 11: 126-36.

159. Yuan H, Wilks MQ, El Fakhri G, Normandin MD, Kaittanis C, Josephson L. Heat-induced-radiolabeling and click chemistry: A powerful combination for generating multifunctional nanomaterials. PloS one. 2017; 12: e0172722.
160. Kaittanis C, Bolaender A, Yoo B, Shah N, Ouerfelli O, Grimm J. Targetable Clinical Nanoparticles for Precision Cancer Therapy Based on Disease-Specific Molecular Inflection Points. Nano Lett. 2017; 17: 7160-8.

161. Zanganeh S, Hutter G, Spitler R, Lenkov O, Mahmoudi M, Shaw A, et al. Iron oxide nanoparticles inhibit tumour growth by inducing pro-inflammatory macrophage polarization in tumour tissues. Nature nanotechnology. 2016; 11: 986-94.

162. Laskar A, Eilertsen J, Li W, Yuan XM. SPION primes THP1 derived M2 macrophages towards M1-like macrophages. Biochemical and biophysical research communications. 2013; 441: 737-42.

163. Janic B, Iskander AS, Rad AM, Soltanian-Zadeh H, Arbab AS. Effects of ferumoxides-protamine sulfate labeling on immunomodulatory characteristics of macrophage-like THP-1 cells. PloS one. 2008; 3: e2499.

164. Muller K, Skepper JN, Posfai M, Trivedi R, Howarth S, Corot C, et al. Effect of ultrasmall superparamagnetic iron oxide nanoparticles (Ferumoxtran-10) on human monocyte-macrophages in vitro. Biomaterials. 2007; 28: 1629-42.

165. Costa da Silva M, Breckwoldt MO, Vinchi F, Correia MP, Stojanovic A, Thielmann CM, et al. Iron Induces Anti-tumor Activity in Tumor-Associated Macrophages. Frontiers in immunology. 2017; 8: 1479

166. Sohail A, Ahmad Z, Beg OA, Arshad S, Sherin L. A review on hyperthermia via nanoparticle-mediated therapy. Bull Cancer. 2017; 104: 452-61.

167. Mertz D, Sandre O, Begin-Colin S. Drug releasing nanoplatforms activated by alternating magnetic fields. Biochim Biophys Acta. 2017; 1861: 1617-41.

168. Golovin YI, Gribanovsky SL, Golovin DY, Klyachko NL, Majouga AG, Master capital A C, et al. Towards nanomedicines of the future: Remote magneto-mechanical actuation of nanomedicines by alternating magnetic fields. J Control Release. 2015; 219: 43-60.

169. Kang H, Kim S, Wong DSH, Jung HJ, Lin S, Zou K, et al. Remote Manipulation of Ligand Nano-Oscillations Regulates Adhesion and Polarization of Macrophages in Vivo. Nano Lett. 2017; 17: 6415-27.

170. Chávez-Galán L Olleros ML, Vesin D, Garcia I Much More than M1 and M2 Macrophages, There are also CD169(+) and TCR(+) Macrophages. Frontiers in immunology. 2015; 6: 263.

171. He C, Carter AB. The Metabolic Prospective and Redox Regulation of Macrophage Polarization. J Clin Cell Immunol. 2015; 6.

172. Roszer T. Understanding the Mysterious M2 Macrophage through Activation Markers and Effector Mechanisms. Mediators of Inflammation. 2015; 2015: 16.

173. Sica A, Bronte V. Altered macrophage differentiation and immune dysfunction in tumor development. Journal of Clinical Investigation. 2007; 117: $1155-66$

174. Anselmo AC, Mitragotri S. Nanoparticles in the clinic. Bioengineering \& Translational Medicine. 2016; 1: 10-29. 